DESY-02-029

March 2002

\title{
Measurement of the $Q^{2}$ and energy dependence of diffractive interactions at HERA
}

\begin{abstract}
ZEUS Collaboration
Abstract

Diffractive dissociation of virtual photons, $\gamma^{*} p \rightarrow X p$, has been studied in $e p$ interactions with the ZEUS detector at HERA. The data cover photon virtualities $0.17<Q^{2}<0.70 \mathrm{GeV}^{2}$ and $3<Q^{2}<80 \mathrm{GeV}^{2}$ with $3<M_{X}<38 \mathrm{GeV}$, where $M_{X}$ is the mass of the hadronic final state. Diffractive events were selected by two methods: the first required the detection of the scattered proton in the ZEUS leading proton spectrometer (LPS); the second was based on the distribution of $M_{X}$. The integrated luminosities of the low- and high- $Q^{2}$ samples used in the LPS-based analysis are $\simeq 0.9 \mathrm{pb}^{-1}$ and $\simeq 3.3 \mathrm{pb}^{-1}$, respectively. The sample used for the $M_{X^{-}}$based analysis corresponds to an integrated luminosity of $\simeq 6.2 \mathrm{pb}^{-1}$. The dependence of the diffractive cross section on $W$, the virtual photon-proton centre-of-mass energy, and on $Q^{2}$ is studied. In the low- $Q^{2}$ range, the energy dependence is compatible with Regge theory and is used to determine the intercept of the Pomeron trajectory. The $W$ dependence of the diffractive cross section exhibits no significant change from the low- $Q^{2}$ to the high- $Q^{2}$ region. In the low- $Q^{2}$ range, little $Q^{2}$ dependence is found, a significantly different behaviour from the rapidly falling cross section measured for $Q^{2}>3 \mathrm{GeV}^{2}$. The ratio of the diffractive to the virtual photon-proton total cross section is studied as a function of $W$ and $Q^{2}$. Comparisons are made with a model based on perturbative QCD.
\end{abstract}




\section{The ZEUS Collaboration}

S. Chekanov, D. Krakauer, S. Magill, B. Musgrave, A. Pellegrino, J. Repond, R. Yoshida Argonne National Laboratory, Argonne, Illinois 60439-4815 ${ }^{n}$

M.C.K. Mattingly

Andrews University, Berrien Springs, Michigan 49104-0380

P. Antonioli, G. Anzivino ${ }^{1}$, G. Bari, M. Basile, L. Bellagamba, D. Boscherini, A. Bruni, G. Bruni, G. Cara Romeo, M. Chiarini, L. Cifarelli, F. Cindolo, A. Contin, M. Corradi, S. De Pasquale, P. Giusti, G. Iacobucci, G. Levi, A. Margotti, T. Massam, R. Nania, C. Nemoz $^{2}$, F. Palmonari, A. Pesci, G. Sartorelli, Y. Zamora Garcia ${ }^{3}$, A. Zichichi University and INFN Bologna, Bologna, Italy ${ }^{e}$

G. Aghuzumtsyan, D. Bartsch, I. Brock, J. Crittenden ${ }^{4}$, S. Goers, H. Hartmann, E. Hilger, P. Irrgang, H.-P. Jakob, A. Kappes, U.F. Katz ${ }^{5}$, R. Kerger ${ }^{6}$, O. Kind, E. Paul, J. Rautenberg ${ }^{7}$, R. Renner, H. Schnurbusch, A. Stifutkin, J. Tandler, K.C. Voss, A. Weber

Physikalisches Institut der Universität Bonn, Bonn, Germany ${ }^{b}$

D.S. Bailey ${ }^{8}$, N.H. Brook ${ }^{8}$, J.E. Cole, B. Foster, G.P. Heath, H.F. Heath, S. Robins, E. Rodrigues ${ }^{9}$, J. Scott, R.J. Tapper, M. Wing H.H. Wills Physics Laboratory, University of Bristol, Bristol, United Kingdom ${ }^{m}$

R. Ayad $^{10}$, M. Capua, L. Iannotti ${ }^{11}$, A. Mastroberardino, M. Schioppa, G. Susinno Calabria University, Physics Department and INFN, Cosenza, Italy ${ }^{e}$

J.Y. Kim, Y.K. Kim, J.H. Lee, I.T. Lim, M.Y. Pac ${ }^{12}$

Chonnam National University, Kwangju, Korea ${ }^{g}$

A. Caldwell, M. Helbich, X. Liu, B. Mellado, S. Paganis, W.B. Schmidke, F. Sciulli Nevis Laboratories, Columbia University, Irvington on Hudson, New York 10027º

J. Chwastowski, A. Eskreys, J. Figiel, K. Olkiewicz, M.B. Przybycień13 ${ }^{13}$, P. Stopa, L. Zawiejski

Institute of Nuclear Physics, Cracow, Poland ${ }^{i}$

B. Bednarek, I. Grabowska-Bold, K. Jeleń, D. Kisielewska, A.M. Kowal, M. Kowal, T. Kowalski, B. Mindur, M. Przybycień, E. Rulikowska-Zarȩbska, L. Suszycki, D. Szuba, J. Szuba ${ }^{14}$

Faculty of Physics and Nuclear Techniques, University of Mining and Metallurgy, Cracow, Poland ${ }^{p}$

A. Kotański ${ }^{15}$, W. Słomiński ${ }^{16}$

Department of Physics, Jagellonian University, Cracow, Poland 
L.A.T. Bauerdick ${ }^{17}$, U. Behrens, K. Borras, V. Chiochia, D. Dannheim, M. Derrick ${ }^{18}$, G. Drews, J. Fourletova, A. Fox-Murphy, U. Fricke, A. Geiser, F. Goebel, P. Göttlicher ${ }^{19}$, O. Gutsche, T. Haas, W. Hain, G.F. Hartner, S. Hillert, U. Kötz, H. Kowalski ${ }^{20}$, H. Labes, D. Lelas, B. Löhr, R. Mankel, M. Martínez ${ }^{17}$, M. Moritz, D. Notz, I.-A. Pellmann, M.C. Petrucci, A. Polini, U. Schneekloth, F. Selonke ${ }^{21}$, B. Surrow ${ }^{22}$, H. Wessoleck, R. Wichmann ${ }^{23}$, G. Wolf, C. Youngman, W. Zeuner

Deutsches Elektronen-Synchrotron DESY, Hamburg, Germany

A. Lopez-Duran Viani, A. Meyer, S. Schlenstedt

DESY Zeuthen, Zeuthen, Germany

G. Barbagli, E. Gallo, C. Genta, P. G. Pelfer

University and INFN, Florence, Italy ${ }^{e}$

A. Bamberger, A. Benen, N. Coppola, P. Markun, H. Raach, S. Wölfle

Fakultät für Physik der Universität Freiburg i.Br., Freiburg i.Br., Germany ${ }^{b}$

M. Bell, P.J. Bussey, A.T. Doyle, C. Glasman, S. Hanlon, S.W. Lee, A. Lupi, G.J. McCance, D.H. Saxon, I.O. Skillicorn

Department of Physics and Astronomy, University of Glasgow, Glasgow, United Kingdom $^{m}$

I. Gialas

Department of Engineering in Management and Finance, Univ. of Aegean, Greece

B. Bodmann, T. Carli, U. Holm, K. Klimek, E. Lohrmann, M. Milite, H. Salehi, S. Stonjek ${ }^{24}$, K. Wick, A. Ziegler, Ar. Ziegler

Hamburg University, Institute of Exp. Physics, Hamburg, Germany ${ }^{b}$

C. Collins-Tooth, C. Foudas, R. Gonçalo ${ }^{9}$, K.R. Long, F. Metlica, D.B. Miller, A.D. Tapper, R. Walker

Imperial College London, High Energy Nuclear Physics Group, London, United Kingdom ${ }^{m}$

P. Cloth, D. Filges

Forschungszentrum Jülich, Institut für Kernphysik, Jülich, Germany

M. Kuze, K. Nagano, K. Tokushuku ${ }^{25}$, S. Yamada, Y. Yamazaki

Institute of Particle and Nuclear Studies, KEK, Tsukuba, Japan ${ }^{f}$

A.N. Barakbaev, E.G. Boos, N.S. Pokrovskiy, B.O. Zhautykov

Institute of Physics and Technology of Ministry of Education and Science of Kazakhstan, Almaty, Kazakhstan

H. Lim, D. Son

Kyungpook National University, Taegu, Korea ${ }^{g}$ 
F. Barreiro, O. González, L. Labarga, J. del Peso, I. Redondo ${ }^{26}$, J. Terrón, M. Vázquez Departamento de Física Teórica, Universidad Autónoma Madrid,Madrid, Spain ${ }^{l}$

M. Barbi, A. Bertolin, F. Corriveau, A. Ochs, S. Padhi, D.G. Stairs, M. St-Laurent

Department of Physics, McGill University, Montréal, Québec, Canada H3A 2T8 ${ }^{a}$

T. Tsurugai

Meiji Gakuin University, Faculty of General Education, Yokohama, Japan

A. Antonov, V. Bashkirov ${ }^{27}$, P. Danilov, B.A. Dolgoshein, D. Gladkov, V. Sosnovtsev, S. Suchkov

Moscow Engineering Physics Institute, Moscow, Russia ${ }^{j}$

R.K. Dementiev, P.F. Ermolov, Yu.A. Golubkov, I.I. Katkov, L.A. Khein, I.A. Korzhavina, V.A. Kuzmin, B.B. Levchenko, O.Yu. Lukina, A.S. Proskuryakov, L.M. Shcheglova, N.N. Vlasov, S.A. Zotkin

Moscow State University, Institute of Nuclear Physics, Moscow, Russia ${ }^{k}$

C. Bokel, J. Engelen, S. Grijpink, E. Koffeman, P. Kooijman, E. Maddox, S. Schagen, E. Tassi, H. Tiecke, N. Tuning, J.J. Velthuis, L. Wiggers, E. de Wolf

NIKHEF and University of Amsterdam, Amsterdam, Netherlands ${ }^{h}$

N. Brümmer, B. Bylsma, L.S. Durkin, J. Gilmore, C.M. Ginsburg, C.L. Kim, T.Y. Ling Physics Department, Ohio State University, Columbus, Ohio $43210^{n}$

S. Boogert, A.M. Cooper-Sarkar, R.C.E. Devenish, J. Ferrando, G. Grzelak, T. Matsushita, M. Rigby, O. Ruske ${ }^{28}$, M.R. Sutton, R. Walczak

Department of Physics, University of Oxford, Oxford United Kingdom ${ }^{m}$

R. Brugnera, R. Carlin, F. Dal Corso, S. Dusini, A. Garfagnini, S. Limentani, A. Longhin, A. Parenti, M. Posocco, L. Stanco, M. Turcato

Dipartimento di Fisica dell' Università and INFN, Padova, Italy ${ }^{e}$

L. Adamczyk ${ }^{29}$, E.A. Heaphy, B.Y. Oh, P.R.B. Saull ${ }^{29}$, J.J. Whitmore ${ }^{30}$

Department of Physics, Pennsylvania State University, University Park, Pennsylvania $16802^{\circ}$

Y. Iga

Polytechnic University, Sagamihara, Japan ${ }^{f}$

G. D'Agostini, G. Marini, A. Nigro

Dipartimento di Fisica, Università 'La Sapienza' and INFN, Rome, Italy ${ }^{e}$

C. Cormack, J.C. Hart, N.A. McCubbin

Rutherford Appleton Laboratory, Chilton, Didcot, Oxon, United Kingdom ${ }^{m}$ 
E. Barberis ${ }^{31}$, C. Heusch, W. Lockman, J.T. Rahn, H.F.-W. Sadrozinski, A. Seiden, D.C. Williams

University of California, Santa Cruz, California $95064^{n}$

I.H. Park

Seoul National University, Seoul, Korea

N. Pavel

Fachbereich Physik der Universität-Gesamthochschule Siegen, Germany

H. Abramowicz, S. Dagan, A. Gabareen, S. Kananov, A. Kreisel, A. Levy

Raymond and Beverly Sackler Faculty of Exact Sciences, School of Physics, Tel-Aviv University, Tel-Aviv, Israel ${ }^{d}$

T. Abe, T. Fusayasu, T. Kohno, K. Umemori, T. Yamashita

Department of Physics, University of Tokyo, Tokyo, Japan ${ }^{f}$

R. Hamatsu, T. Hirose, M. Inuzuka, S. Kitamura ${ }^{32}$, K. Matsuzawa, T. Nishimura

Tokyo Metropolitan University, Deptartment of Physics, Tokyo, Japan ${ }^{f}$

M. Arneodo ${ }^{33}$, N. Cartiglia, R. Cirio, M. Costa, M.I. Ferrero, L. Lamberti ${ }^{34}$, S. Maselli, V. Monaco, C. Peroni, M. Ruspa, R. Sacchi, A. Solano, A. Staiano

Università di Torino, Dipartimento di Fisica Sperimentale and INFN, Torino, Italy ${ }^{e}$

R. Galea, T. Koop, G.M. Levman, J.F. Martin, A. Mirea, A. Sabetfakhri

Department of Physics, University of Toronto, Toronto, Ontario, Canada M5S $1 A 7^{a}$

J.M. Butterworth, C. Gwenlan, R. Hall-Wilton, T.W. Jones, J.B. Lane, M.S. Lightwood, J.H. Loizides ${ }^{35}$, B.J. West

Physics and Astronomy Department, University College London, London, United Kingdom $^{m}$

J. Ciborowski ${ }^{36}$, R. Ciesielski ${ }^{37}$, R.J. Nowak, J.M. Pawlak, B. Smalska ${ }^{38}$, J. Sztuk ${ }^{39}$, T. Tymieniecka ${ }^{40}$, A. Ukleja ${ }^{40}$, J. Ukleja, J.A. Zakrzewski, A.F. Żarnecki

Warsaw University, Institute of Experimental Physics, Warsaw, Poland ${ }^{q}$

M. Adamus, P. Plucinski

Institute for Nuclear Studies, Warsaw, Poland ${ }^{q}$

Y. Eisenberg, L.K. Gladilin ${ }^{41}$, D. Hochman, U. Karshon

Department of Particle Physics, Weizmann Institute, Rehovot, Israel ${ }^{c}$

D. Kçira, S. Lammers, L. Li, D.D. Reeder, A.A. Savin, W.H. Smith

Department of Physics, University of Wisconsin, Madison, Wisconsin $53706^{n}$

A. Deshpande, S. Dhawan, V.W. Hughes, P.B. Straub

Department of Physics, Yale University, New Haven, Connecticut 06520-8121 ${ }^{n}$ 
S. Bhadra, C.D. Catterall, S. Fourletov, S. Menary, M. Soares, J. Standage

Department of Physics, York University, Ontario, Canada M3J 1P3 ${ }^{a}$ 
${ }^{1}$ now at Università di Perugia, Dipartimento di Fisica, Perugia, Italy

2 now at E.S.R.F., Grenoble, France

3 now at Inter American Development Bank, Washington DC, USA

${ }^{4}$ now at Cornell University, Ithaca, NY, USA

${ }^{5}$ on leave of absence at University of Erlangen-Nürnberg, Germany

${ }^{6}$ now at Ministère de la Culture, de L'Enseignement Supérieur et de la Recherche, Luxembourg

${ }^{7}$ supported by the GIF, contract I-523-13.7/97

8 PPARC Advanced fellow

${ }^{9}$ supported by the Portuguese Foundation for Science and Technology (FCT)

10 now at Temple University, Philadelphia, PA, USA

11 now at Consoft Sistemi, Torino, Italy

12 now at Dongshin University, Naju, Korea

13 now at Northwestern Univ., Evanston, IL, USA

14 partly supported by the Israel Science Foundation and the Israel Ministry of Science

15 supported by the Polish State Committee for Scientific Research, grant. no. 2P03B 09322

16 member of Dept. of Computer Science, supported by the Polish State Committee for Sci. Res., grant no. 2P03B 06116

17 now at Fermilab, Batavia, IL, USA

18 on leave from Argonne National Laboratory, USA

19 now at DESY group FEB

${ }^{20}$ on leave of absence at Columbia Univ., Nevis Labs., N.Y., USA

21 retired

22 now at Brookhaven National Lab., Upton, NY, USA

23 now at Mobilcom AG, Rendsburg-Büdelsdorf, Germany

24 supported by NIKHEF, Amsterdam, NL

25 also at University of Tokyo

26 now at LPNHE Ecole Polytechnique, Paris, France

27 now at Loma Linda University, Loma Linda, CA, USA

28 now at IBM Global Services, Frankfurt/Main, Germany

29 partly supported by Tel Aviv University

30 on leave of absence at The National Science Foundation, Arlington, VA, USA

31 now at Lawrence Berkeley National Laboratory, Berkeley, CA, USA

32 present address: Tokyo Metropolitan University of Health Sciences, Tokyo 116-8551, Japan

33 also at Università del Piemonte Orientale, Novara, Italy

34 now at Università di Torino, Dipartimento di Medicina Interna, Torino, Italy

35 supported by Argonne National Laboratory, USA

36 also at Łódź University, Poland

37 supported by the Polish State Committee for Scientific Research, grant no. 2 P03B 07222 
${ }^{38}$ supported by the Polish State Committee for Scientific Research, grant no. 2 P03B 00219

${ }^{39}$ Łódź University, Poland

${ }^{40}$ sup. by Pol. State Com. for Scien. Res., 5 P03B 09820 and by Germ. Fed. Min. for Edu. and Research (BMBF), POL 01/043

${ }^{41}$ on leave from MSU, partly supported by University of Wisconsin via the U.S.-Israel BSF 
a supported by the Natural Sciences and Engineering Research Council of Canada (NSERC)

$b$ supported by the German Federal Ministry for Education and Research (BMBF), under contract numbers HZ1GUA 2, HZ1GUB 0, HZ1PDA 5, HZ1VFA 5

${ }^{c}$ supported by the MINERVA Gesellschaft für Forschung GmbH, the Israel Science Foundation, the U.S.-Israel Binational Science Foundation, the Israel Ministry of Science and the Benozyio Center for High Energy Physics

$d$ supported by the German-Israeli Foundation, the Israel Science Foundation, and by the Israel Ministry of Science

$e$ supported by the Italian National Institute for Nuclear Physics (INFN)

$f$ supported by the Japanese Ministry of Education, Science and Culture (the Monbusho) and its grants for Scientific Research

$g \quad$ supported by the Korean Ministry of Education and Korea Science and Engineering Foundation

$h$ supported by the Netherlands Foundation for Research on Matter (FOM)

$i$ supported by the Polish State Committee for Scientific Research, grant no. 620/E-77/SPUB-M/DESY/P-03/DZ 247/2000-2002

$j$ partially supported by the German Federal Ministry for Education and Research (BMBF)

$k$ supported by the Fund for Fundamental Research of Russian Ministry for Science and Education and by the German Federal Ministry for Education and Research (BMBF)

$l$ supported by the Spanish Ministry of Education and Science through funds provided by CICYT

$m$ supported by the Particle Physics and Astronomy Research Council, UK

$n$ supported by the US Department of Energy

$o$ supported by the US National Science Foundation

$p$ supported by the Polish State Committee for Scientific Research, grant no. 112/E-356/SPUB-M/DESY/P-03/DZ 301/2000-2002, 2 P03B 13922

$q$ supported by the Polish State Committee for Scientific Research, grant no. 115/E-343/SPUB-M/DESY/P-03/DZ 121/2001-2002, 2 P03B 07022 


\section{Introduction}

The properties of high-energy hadron-hadron cross sections, notably the energy dependence of the total and elastic cross sections, are described successfully by Regge phenomenology in terms of the exchange of the Pomeron trajectory, $\alpha_{\mathbb{P}}(t)=\alpha_{\mathbb{P}}(0)+\alpha_{\mathbb{P}}^{\prime} t$, where $t$ is the squared four-momentum carried by the exchange [1]. The intercept and slope of the trajectory were found to be $\alpha_{\mathbb{P}}(0)=1.08$ and $\alpha_{\mathbb{P}}^{\prime}=0.25 \mathrm{GeV}^{-2}$, respectively, by Donnachie and Landshoff [2] using the energy dependence of the hadron-hadron total and elastic cross sections. Such a Pomeron trajectory is referred to as "the soft Pomeron". At high energies, hadron-hadron total cross sections, including the $\gamma p$ total cross section, can be expressed in terms of this trajectory as

$$
\sigma \propto\left(W^{2}\right)^{\alpha_{\mathbb{P}}(0)-1}
$$

where $W$ is the virtual photon-proton centre-of-mass energy.

Measurements of the diffractive dissociation of photons have shown that, for quasi-real photons $\left(Q^{2} \approx 0\right.$, photoproduction, where $Q^{2}$ is the exchanged photon virtuality), the value of $\alpha_{I P}(0)$ is compatible with the expectations based on soft-Pomeron exchange [3, [4]. The study of diffractive processes in ep collisions at large virtualities has opened up the possibility of investigating the Pomeron in a regime where perturbative QCD (pQCD) is applicable [5]. In this regime, the exchange of the Pomeron trajectory may be described, at lowest order, as two-gluon exchange in the $t$ channel so that the cross section is proportional to the square of the gluon density in the proton. Since the gluon distribution rises steeply at small Bjorken $x$ (or, equivalently, for large values of $W$ ), a possible signature of the transition from the soft non-perturbative regime to the hard perturbative regime is a change to a $W$-dependence of the cross section steeper than that from the exchange of a soft-Pomeron trajectory. The value of the Pomeron intercept, $\alpha_{I P}(0)$, measured in the deep inelastic scattering (DIS) regime $\left(Q^{2} \gtrsim\right.$ a few $\left.\mathrm{GeV}^{2}\right)$ is larger than that of the soft Pomeron [6, 7], which suggests that pQCD effects have become important.

In analogy with the usual DIS formalism for the proton structure function, $F_{2}$, one can introduce a diffractive structure function, $F_{2}^{D}$. Studies of photon diffractive-dissociation have shown that, for $Q^{2} \gtrsim 1 \mathrm{GeV}^{2}, F_{2}^{D}$ has only a weak, logarithmic, dependence on

$Q^{2}$ [6,7]. However, conservation of the electromagnetic current requires that both $F_{2}^{D}$ and $F_{2}$ must behave like $Q^{2}$ as $Q^{2} \rightarrow 0$.

In this paper, the inclusive diffractive dissociation of virtual photons, $\gamma^{*} p \rightarrow X p$, is investigated by studying the reaction $e p \rightarrow e X p$ at HERA both in the perturbative region $\left(Q^{2} \gg 1 \mathrm{GeV}^{2}\right)$ and in the transition region between the non-perturbative $\left(Q^{2} \sim 0\right)$ and perturbative regions. The measurements are presented as a function of $W$ and $Q^{2}$. The Pomeron intercept is determined through the measurement of the energy dependence of the diffractive cross section in the transition region, which has not previously been 
explored in diffraction. The $W$ and $Q^{2}$ behaviour of the diffractive cross section and of the virtual photon-proton total cross section, $\sigma_{\text {tot }}^{\gamma^{*} p}$, are compared by studying their ratio as a function of $W$ and $Q^{2}$.

Diffractive events were selected by two methods. The first required the detection of the scattered proton in the ZEUS leading proton spectrometer (LPS) and is referred to as the "LPS method". Although statistically limited because of the small acceptance of the LPS, this method permits the selection of events with negligible background from the double-dissociative reaction, $e p \rightarrow e X N$, where the proton also diffractively dissociates into a state $N$ of mass $M_{N}$ that escapes undetected in the beam pipe. The LPS method also gives access to higher values of $M_{X}$, the mass of the hadronic final-state system, $X$, and allows the measurement of the squared four-momentum transfer at the proton vertex, $t$. The second method, henceforth referred to as the " $M_{X}^{2}$ method" [0], is based on the characteristics of the distribution of $M_{X}$. The sample selected with the $M_{X}^{2}$ method contains a background contribution from the double-dissociative events.

This paper presents results in the region $0.17<Q^{2}<0.70 \mathrm{GeV}^{2}$, obtained with both methods, and in the region $3<Q^{2}<80 \mathrm{GeV}^{2}$ using only the LPS. The measurements cover the region $3<M_{X}<38 \mathrm{GeV}$. Results in the DIS region obtained using the $M_{X}^{2}$ method have been previously reported [7].

\section{Kinematic variables and cross sections}

Inclusive diffractive dissociation of virtual photons in positron-proton collisions, ep $\rightarrow$ $e X p$, can be described by the kinematic variables $Q^{2}, W, M_{X}$, and $t$. The differential cross section for $\gamma^{*} p \rightarrow X p$ is related to the cross section for the reaction $e p \rightarrow e X p$ by [8]

$$
\frac{d^{4} \sigma_{\text {diff }}^{e p}\left(Q^{2}, W, M_{X}, t\right)}{d \ln Q^{2} d \ln W d M_{X} d t}=\Gamma\left(Q^{2}, W\right) \frac{d^{2} \sigma_{\text {diff }}^{\gamma^{*} p}\left(Q^{2}, W, M_{X}, t\right)}{d M_{X} d t}
$$

where

$$
\Gamma=\frac{\alpha}{\pi}\left[1+(1-y)^{2}\right]
$$

is the virtual photon flux, $\alpha$ is the fine-structure constant, $y \simeq\left(W^{2}+Q^{2}\right) / s$ is the fraction of the positron energy transferred to the proton in its rest frame, and $s$ is the square of the positron-proton centre-of-mass energy.

In analogy with the formalism of inclusive deep inelastic ep scattering, the diffractive cross section for the reaction $e p \rightarrow e X p$ can also be expressed in terms of diffractive structure functions [9]:

$$
\frac{d^{4} \sigma_{\mathrm{diff}}^{\mathrm{ep}}}{d \beta d Q^{2} d x_{\mathbb{P}} d t}=\frac{4 \pi \alpha^{2}}{\beta Q^{4}}\left\{1-y+\frac{y^{2}}{2\left(1+R^{D(4)}\left(\beta, Q^{2}, x_{\mathbb{P}}, t\right)\right)}\right\} F_{2}^{D(4)}\left(\beta, Q^{2}, x_{\mathbb{P}}, t\right),
$$


where the diffractive structure function $F_{2}^{D(4)}$ and the ratio of the cross sections for longitudinal and transverse photons, $R^{D(4)}$, have been introduced.

The variables $x_{\mathbb{P}}$ and $\beta$ are related to $Q^{2}, W^{2}, M_{X}^{2}$ and $t$ by

$$
\begin{aligned}
x_{\mathbb{P}} & =\frac{Q^{2}+M_{X}^{2}-t}{Q^{2}+W^{2}-M_{p}^{2}}, \\
\beta & =\frac{Q^{2}}{Q^{2}+M_{X}^{2}-t},
\end{aligned}
$$

where $M_{p}$ is the proton mass. The variables $x_{\mathbb{P}}$ and $\beta$ can be interpreted, assuming the $t$-channel exchange of a Pomeron with partonic structure, as the fraction of the proton momentum carried by the Pomeron and the fraction of the Pomeron momentum carried by the struck parton, respectively.

Equations (11) and (21) can be combined to give

$$
\frac{d^{2} \sigma_{\text {diff }}^{\gamma^{*} p}}{d M_{X} d t}=\frac{W^{2}}{Q^{2}+W^{2}} \frac{2 M_{X}}{Q^{2}+M_{X}^{2}} \frac{4 \pi^{2} \alpha}{Q^{2}} x_{\mathbb{P}} F_{2}^{D(4)}
$$

where $|t| \ll Q^{2}+M_{X}^{2}$ has been assumed and $M_{p}$ and $R^{D(4)}$ have been neglected [10. An analogous expression holds for the three-fold differential diffractive structure function, $F_{2}^{D(3)}$, obtained by integrating $F_{2}^{D(4)}$ over $t$. Equation (3) is the diffractive analogue of the expression $\sigma_{\text {tot }}^{\gamma^{*} p}=\left(4 \pi^{2} \alpha / Q^{2}\right) F_{2}$ which holds for inclusive $\gamma^{*} p$ scattering at high $W$.

\section{$3 \quad$ Experimental set-up}

The measurements were performed at the HERA ep collider at DESY between 1995 and 1997 using the ZEUS detector. At that time, HERA operated at a proton energy of $820 \mathrm{GeV}$ and a positron energy of $27.5 \mathrm{GeV}$.

A detailed description of the ZEUS detector can be found elsewhere [11. A brief outline of the components most relevant for this analysis is given below.

Charged particles are tracked by the central tracking detector (CTD) [12], which operates in a magnetic field of $1.43 \mathrm{~T}$ provided by a thin superconducting coil. The CTD consists of 72 cylindrical drift-chamber layers, organised in 9 superlayers covering the polar-angle円 region $15^{\circ}<\theta<164^{\circ}$. The relative transverse-momentum resolution for full-length tracks is $\sigma\left(p_{t}\right) / p_{t}=0.0058 p_{t} \oplus 0.0065 \oplus 0.0014 / p_{t}$, with $p_{t}$ in $\mathrm{GeV}$.

1 The ZEUS coordinate system is a right-handed Cartesian system, with the $Z$ axis pointing in the proton-beam direction, referred to as the "forward direction", and the $X$ axis pointing left towards the centre of HERA. The coordinate origin is at the nominal interaction point. The pseudorapidity is defined as $\eta=-\ln \left(\tan \frac{\theta}{2}\right)$, where the polar angle, $\theta$, is measured with respect to the proton-beam direction. 
The high-resolution uranium-scintillator calorimeter (CAL) [13 consists of three parts: the forward (FCAL), the barrel (BCAL) and the rear (RCAL) calorimeters. Each part is subdivided transversely into towers and longitudinally into one electromagnetic section (EMC) and either one (in RCAL) or two (in FCAL and BCAL) hadronic sections (HAC). The smallest subdivision of the calorimeter is called a cell. The CAL relative energy resolutions, as measured under test-beam conditions, are $\sigma(E) / E=0.18 / \sqrt{E}$ for electrons and $\sigma(E) / E=0.35 / \sqrt{E}$ for hadrons $(E$ in $\mathrm{GeV})$.

Low- $Q^{2}$ events $\left(0.17<Q^{2}<0.70 \mathrm{GeV}^{2}\right)$ were tagged by requiring the identification of the scattered positron in the beam pipe calorimeter (BPC) [14, 15. The BPC was a tungstenscintillator sampling calorimeter, located close to the beam pipe, $3 \mathrm{~m}$ downstream of the interaction point in the positron-beam direction. The relative energy resolution from test-beam results was $\sigma(E) / E=0.17 / \sqrt{E}(E$ in $\mathrm{GeV})$. Each scintillator layer consisted of $8 \mathrm{~mm}$-wide strips. Using the logarithmically weighted shower position, the impact position of the scattered positron could be measured with an accuracy of about $1 \mathrm{~mm}$. For events with $Q^{2}>3 \mathrm{GeV}^{2}$, the impact point of the scattered positron was determined with the small-angle rear tracking detector (SRTD) [16 or the CAL. The SRTD is attached to the front face of the RCAL and consists of two planes of scintillator strips, $1 \mathrm{~cm}$ wide and $0.5 \mathrm{~cm}$ thick, arranged in orthogonal orientations and read out via optical fibres and photomultiplier tubes. It covers a region of about $68 \times 68 \mathrm{~cm}^{2}$ in $X$ and $Y$, excluding a $10 \times 20 \mathrm{~cm}^{2}$ hole at the centre for the beam pipe.

The LPS [17] detected positively charged particles scattered at small angles and carrying a substantial fraction, $x_{L}$, of the incoming proton momentum; these particles remain in the beam pipe and their trajectory was measured by a system of silicon microstrip detectors that can be inserted very close (typically a few $\mathrm{mm}$ ) to the proton beam. The detectors were grouped in six stations, S1 to S6, placed along the beam line in the direction of the proton beam, between $23.8 \mathrm{~m}$ and $90.0 \mathrm{~m}$ from the interaction point. The track deflections induced by the magnets of the proton beam-line allow a momentum analysis of the scattered proton. For the present measurements, only stations S4, S5 and S6 were used. The resolutions were better than $1 \%$ on the longitudinal momentum and $5 \mathrm{MeV}$ on the transverse momentum. The effective transverse-momentum resolution is, however, dominated by the intrinsic transverse-momentum spread of the proton beam at the interaction point, which is about $40 \mathrm{MeV}$ in the horizontal plane and about $100 \mathrm{MeV}$ in the vertical plane.

\section{Reconstruction of the kinematic variables}

Different methods have been used for the reconstruction of the kinematic variables $Q^{2}$ and $W$, depending on the $Q^{2}$ range of the measurement. At low $Q^{2}, 0.17<Q^{2}<0.70 \mathrm{GeV}^{2}$ (hereafter referred to as the "BPC region"), the energy, $E_{e}^{\prime}$, and angle, $\theta_{e}$, of the scattered 
positron measured in the BPC were used ("electron method") to determine the kinematic variables from

$$
\begin{aligned}
Q^{2} & =2 E_{e} E_{e}^{\prime}\left(1+\cos \theta_{e}\right) \\
W & =\sqrt{4 E_{e} E_{p}\left[1-\frac{E_{e}^{\prime}}{2 E_{e}}\left(1-\cos \theta_{e}\right)\right]},
\end{aligned}
$$

where $E_{p}$ and $E_{e}$ represent the proton and positron beam energies, respectively. For $Q^{2}>3 \mathrm{GeV}^{2}$ (the "DIS region"), $Q^{2}$ and $W$ were reconstructed with the double angle method [18] using the energy depositions in the CAL.

For the reconstruction of the mass of the diffractive system $X$, the energy deposits in the CAL and the track momenta measured in the CTD were clustered to form energy-flow objects (EFOs) [0, 19]. The EFOs thus include the information from both neutral and charged particles in an optimal way. The mass, $M_{X}$, was then obtained from the EFOs via

$$
M_{X}=\sqrt{\left(\sum_{i} E_{i}\right)^{2}-\left(\sum_{i} p_{X i}\right)^{2}-\left(\sum_{i} p_{Y i}\right)^{2}-\left(\sum_{i} p_{Z i}\right)^{2}},
$$

where the subscript $i$ denotes an individual EFO; the EFOs associated with the scattered positron are excluded from the sums.

The momentum of those scattered protons detected in the LPS, $p^{\text {LPS }}$, was measured, along with its component perpendicular (parallel) to the beam direction, $p_{T}^{\mathrm{LPS}}\left(p_{Z}^{\mathrm{LPS}}\right)$. From these quantities, the fractional momentum of the scattered proton, $x_{L}$, and $t$ were determined via

$$
\begin{aligned}
x_{L} & =p_{Z}^{\mathrm{LPS}} / E_{p}, \\
t & =-\frac{\left(p_{T}^{\mathrm{LPS}}\right)^{2}}{x_{L}} .
\end{aligned}
$$

Two quantities, $y$ and $\delta \equiv \sum_{i}\left(E-p_{Z}\right)_{i}+E_{e^{\prime}}\left(1-\cos \theta_{e}\right)$, the sum of $E-P_{Z}$ over all final-state particles in the event, were used in the event selection. The former was reconstructed either using the electron method (and denoted by $y_{e}$ ) or from the EFOs using the Jacquet-Blondel estimator 20 as

$$
y_{J B}=\frac{\sum_{i}\left(E-p_{Z}\right)_{i}}{2 E_{e}}
$$

where the sum is over all EFOs, excluding those assigned to the scattered positron. Energy and momentum conservation require $\delta$ to be twice the positron beam energy for a completely measured final state and neglecting resolution effects. 


\section{Monte Carlo simulation}

Monte Carlo (MC) generators were used to determine the acceptance of the apparatus. The reaction $e p \rightarrow e X p$ was simulated in the BPC region with the EPSOFT2.0 [7, 21, 22] MC generator interfaced to HERACLES4.6 [23], which simulates initial- and final-state QED radiation. For the description of the diffractive dissociation of virtual photons, $\gamma^{*} p \rightarrow X p$, EPSOFT uses the triple-Regge formalism [1], in which the inclusive diffractive cross section can be expressed in terms of three trajectories. If all the trajectories are Pomerons (IPIPIP), the cross-section $d \sigma / d M_{X}^{2}$ is approximately proportional to $1 / M_{X}^{2}$. If one of the trajectories is a Reggeon (IPIPIR), the cross-section $d \sigma / d M_{X}^{2}$ falls as $\sim 1 / M_{X}^{3}$. EPSOFT also simulates exclusive vector-meson production, $e p \rightarrow e V p$, where $V=\rho^{0}$, $\omega$ or $\phi$, and non-diffractive $e p$ interactions, $e p \rightarrow e Y$. Production of $J / \psi$ mesons has negligible effects on the acceptance and was not considered. EPSOFT was also used to simulate the double-dissociative reaction, $e p \rightarrow e X N$, where the proton diffractively dissociates into the state $N$.

The second generator, used for the DIS region, was RAPGAP2.06 [24], where, for the diffractive structure function, a factorisable expression was assumed based on the model of Ingelman and Schlein [25]. In particular, a superposition of non-interfering Pomeron and sub-leading trajectories was used ("fit B", as determined by the H1 Collaboration [6]) together with the "fit 3" Pomeron parton density functions [6]. Again, initial- and finalstate QED radiation were simulated using HERACLES.

All generated events were passed through the standard ZEUS detector simulation, based on the GEANT3.13 program [26], and the trigger-simulation package.

\section{Identification of the scattered positron}

For the BPC sample, the events were selected in the trigger by requiring the presence of a scattered positron in the BPC. A positron with energy greater than $7 \mathrm{GeV}$ was required in the offline analysis [14, 15]. The following cuts were applied to reduce the contamination from photoproduction events, radiative events, and beam-related background:

- $y_{J B}>0.05$

- $35<\delta<65 \mathrm{GeV}$;

- $\left|Z_{\mathrm{VTX}}\right|<50 \mathrm{~cm}$, where $Z_{\mathrm{VTX}}$ is the $Z$ coordinate of the reconstructed vertex.

For the DIS sample, the events used for the analysis were selected in the trigger by requiring the presence of a scattered positron in the CAL. Offline, a positron in the RCAL 
with energy greater than $10 \mathrm{GeV}$ was required. A positron finder based on a neuralnetwork was used [27]. The following cuts were applied to reduce the contamination from photoproduction events, radiative events, and beam-related background:

- $y_{J B}>0.03$;

- $y_{e}<0.95$;

- $35<\delta<65 \mathrm{GeV}$;

- $-50<Z_{\mathrm{VTX}}<100 \mathrm{~cm}$.

\section{The LPS method}

Diffractive events are characterised by a final state proton scattered at very small angle and with energy nearly equal to that of the incoming proton. In the LPS method, diffractive events are then defined as those having a proton detected in the LPS with $x_{L} \approx 1$. Figure 1(a) shows the measured $x_{L}$ spectrum, uncorrected for acceptance. The diffractive peak is clearly visible for values of $x_{L}$ close to unity. For the present analysis, $x_{L}>0.97$ was required. Previous studies [28] indicate that the double-dissociative contribution to such events is negligible.

Two data samples, collected in 1995, were analysed with the LPS method. The BPC sample, corresponding to a luminosity of $0.90 \pm 0.01 \mathrm{pb}^{-1}$, covers the range $0.17<Q^{2}<$ $0.70 \mathrm{GeV}^{2}$ and $90<W<250 \mathrm{GeV}$. The DIS sample covers the region $3<Q^{2}<80 \mathrm{GeV}^{2}$ and $80<W<250 \mathrm{GeV}$, and corresponds to a luminosity of $3.30 \pm 0.03 \mathrm{pb}^{-1}$. The analysis was limited to the range $3<M_{X}<38 \mathrm{GeV}$ for the $\mathrm{BPC}$ sample and $3<M_{X}<33 \mathrm{GeV}$ for the DIS sample.

The candidate proton was tracked along the proton beam line and was rejected if, at any point, the reconstructed minimum distance of approach to the beam pipe, $\Delta_{\text {pipe }}$, was less than $400 \mu \mathrm{m}$ or if the distance to the edge of the sensitive region of any LPS station, $\Delta_{\text {plane }}$, was smaller than $200 \mu \mathrm{m}$. These cuts reduce the sensitivity of the acceptance to the uncertainty in the position of the beam-pipe apertures and of the detector edges. In addition, $t$ was required to be in the region $0.073<|t|<0.4 \mathrm{GeV}^{2}$, where the LPS acceptance is well understood [17]. Beam-halo background results from a scattered proton, with energy close to that of the beam, originating from an interaction of a beam proton with the residual gas or with the beam collimators. In this case, the proton measured in the LPS is uncorrelated with the activity in the central ZEUS detector. This background was suppressed by the requirement that the sum of the energy and the longitudinal component of the total momentum measured in the CAL, the BPC and the LPS be less than the kinematic limit of $2 E_{p}:\left(E+p_{Z}\right)_{\mathrm{CAL}}+\left(E+p_{Z}\right)_{\mathrm{BPC}}+2 p_{Z}^{\mathrm{LPS}}<1665 \mathrm{GeV}$. This cut takes 
into account the resolution of the measurement of $p_{Z}^{\text {LPS }}$. A residual beam-halo background of approximately $8 \%$, remaining after the cut, was subtracted statistically.

In the BPC analysis, the measured number of diffractive events was corrected bin-by-bin. From this acceptance-corrected number of events, the cross section for the diffractive dissociation of virtual photons, $\gamma^{*} p \rightarrow X p$, was determined taking into account the integrated luminosity, bin widths, and bin-centring corrections.

In the DIS analysis, the cross section for the diffractive dissociation of virtual photons at a given point within a bin was obtained from the ratio of the measured number of diffractive events to the number of events in that bin predicted from the MC simulation, multiplied by the $\gamma^{*} p \rightarrow X p$ cross section calculated at that point by the Monte Carlo generator. Both the acceptance and the bin-centring corrections were thus taken from the MC simulation.

In both the BPC and DIS analyses, the diffractive cross-section $d \sigma_{\text {diff }}^{\gamma^{*} p} / d M_{X}$ was directly measured only in the region $0.073<|t|<0.4 \mathrm{GeV}^{2}$ and extrapolated to the full $t$ range using the $t$ dependence assumed in the Monte Carlo generator. In the region covered by the present measurements, this is roughly equivalent to carrying out an integration over $t$ assuming an exponential dependence on $t, e^{-b|t|}$, with $b \sim 7.5 \mathrm{GeV}^{-2}$.

\section{The $M_{X}^{2}$ method}

Diffractive photon dissociation, $\gamma^{*} p \rightarrow X p$, is characterised by the exchange of a colourless object, the Pomeron, between the virtual photon and the proton. This suppresses QCD radiation, and hence the production of hadrons, in the rapidity region between the hadronic system $X$ and the scattered proton, yielding a forward rapidity gap, a characteristic feature of diffractive interactions. This feature is reflected in the dependence of the cross section on $M_{X}, d \sigma_{\text {diff }}^{\gamma^{*} p} / d M_{X} \propto 1 / M_{X}^{2 \alpha_{P}(0)-1}$, i.e. approximately flat as a function of $\ln M_{X}^{2}$. In contrast, for non-diffractive events, large rapidity gaps are exponentially suppressed by QCD radiation, which populates the region between the struck quark and the coloured proton remnant. In this case, under the assumption of uniform, random and uncorrelated particle emission in rapidity, the $\ln M_{X}^{2}$ distribution falls exponentially towards low $M_{X}$ values. The different properties of the $\ln M_{X}^{2}$ distribution for diffractive and non-diffractive events are exploited in the $M_{X}^{2}$ method [7].

The $M_{X}^{2}$ method was used to analyse BPC data taken in 1996-97, corresponding to an integrated luminosity of $6.2 \pm 0.1 \mathrm{pb}^{-1}$. The kinematic range used was $0.22<Q^{2}<$ $0.70 \mathrm{GeV}^{2}, 90<W<220 \mathrm{GeV}$ and $3.0<M_{X}<12.2 \mathrm{GeV}$. 


\subsection{Selection of the diffractive signal}

Figure 1(b) shows a representative distribution of $\ln M_{X}^{2}$ for datal in the bin $0.220<Q^{2}<$ $0.324 \mathrm{GeV}^{2}$ and $150<W<180 \mathrm{GeV}$, compared to the distribution of the simulated events generated with EPSOFT. Also shown are the four individual contributions generated with EPSOFT for non-diffractive events, for the IPIPIP and IPIPIR contributions (shown combined in the figure), which lead to the diffractive dissociation of the photon, and for vector-meson production. Diffractive events dominate the region of low $\ln M_{X}^{2}$, while non-diffractive events exhibit a large peak at high $\ln M_{X}^{2}$ and a steep exponential fall-off towards lower $\ln M_{X}^{2}$ values. The relative weights of the four subprocesses are obtained from fits to the $\ln M_{X}^{2}$ distribution of the data. The resulting sum of the MC events (open histogram) from the various subprocesses provides a reasonably good description of the data in the region of interest, $\ln M_{X}^{2}<8.5$.

In the region $\ln M_{X}^{2} \sim 4$, the diffractive contribution to the events in Fig. 1)(b) depends only weakly on $\ln M_{X}^{2}$. The expression

$$
\frac{d N}{d \ln M_{X}^{2}}=D+C \exp \left(B \ln M_{X}^{2}\right)
$$

was therefore fitted to the data and the parameters $D, C$ and $B$ were determined for each $\left(Q^{2}, W\right)$ region. The exponential term (with $B=1.44 \pm 0.02$ ), ascribed to non-diffractive events, was subtracted statistically to obtain the diffractive contribution. The parameter $D$ was thus not used directly to determine the diffractive cross section. The exponential term resulting from the fit to the data of Fig. I(b) is shown in Fig. I(c).

The cross-section measurement was restricted to the range $2.2<\ln M_{X}^{2}<5.0$, corresponding to $3.0<M_{X}<12.2 \mathrm{GeV}$. The lower limit on $M_{X}$ suppresses the contribution from diffractive vector-meson production, while the upper bound was chosen such that the non-diffractive contribution to the higher- $M_{X}$ bins was always less than $50 \%$.

\subsection{Proton-dissociative contribution}

The diffractive sample of $e p \rightarrow e X p$ events selected with the $M_{X}^{2}$ method as discussed in Section 8.1 contains a contribution from the double-dissociative reaction $e p \rightarrow e X N$. The system $N$ escapes undetected through the forward beam pipe, unless the proton dissociates into a state of sufficiently high mass, in which case some of the particles from the system $N$ have transverse momenta large enough that they are detected in the FCAL region around the forward beam pipe. The contribution of the double-dissociative reaction $e p \rightarrow e X N$ was simulated and studied with EPSOFT.

Energy deposits in the FCAL, arising from the proton-dissociative remnant, give rise to a measured value of $M_{X}$ considerably higher than the true value. In such events, there

${ }^{2}$ The data shown in Figs. 1 $1(b, c)$ result from the cuts discussed in Section 8.2 . 
is a gap in rapidity between the FCAL deposits from the proton remnant at high $\eta$ and the system $X$ at lower $\eta$, and the invariant mass of the low- $\eta$ system is small with respect to the measured (apparent) $M_{X}$. Exploiting these features, events were rejected if they fulfilled all of the following three conditions:

- the maximum $\eta\left(\eta_{\mathrm{MAX}}\right)$ of the EFOs was greater than 2.5;

- the maximum rapidity gap between adjacent EFOs was greater than 3.5;

- the mass reconstructed from EFOs with $\eta<2.5$ was less than $0.6 M_{X}$.

These cuts rejected approximately $10 \%$ of the data sample. The simulations using the EPSOFT MC program indicate that about $45 \%$ of these rejected events are from the double-dissociative reaction, $\gamma^{*} p \rightarrow X N$, and the events that survive the cuts consist of photon-dissociative events as well as events from the double-dissociative reaction with $M_{N}<6 \mathrm{GeV}$.

The measured number of events in each $\left(Q^{2}, W, M_{X}\right)$ bin was corrected for acceptance to determine the number of produced events by means of the Singular Value Decomposition (SVD) method [29, which allows the evaluation of error correlations between adjacent bins. The number of events thus obtained was divided by the luminosity and the binwidths to evaluate the average $e p \rightarrow e X p$ three-fold differential cross section for each

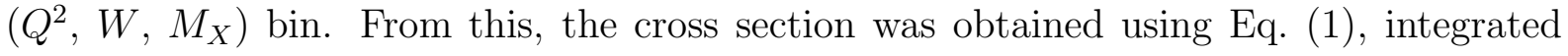
over $t$ and evaluated at the logarithmic centre of the bin. The residual double-dissociative contribution in the data leads to an overestimation of the cross section for the diffractive dissociation of virtual photons, $\gamma^{*} p \rightarrow X p$; it was evaluated using the LPS data and subtracted as discussed in Section 10.

\section{Systematic uncertainties}

The main sources of systematic uncertainty can be classified into three groups: the positron measurement in the BPC or the CAL; the measurement of the hadronic final state in the CAL; and the measurement of the scattered proton in the LPS:

- measurement of the scattered positron:

- for the BPC samples, the effects of the uncertainty in the absolute BPC energy scale [15] $( \pm 0.5 \%)$, the positron-selection criteria and the alignment of the BPC result in an uncertainty in the cross section that is typically $\pm 7 \%$ and always smaller than $\pm 20 \%$; 
- for the DIS sample, the $10 \mathrm{GeV}$ cut on the scattered-positron energy was changed by $\pm 2 \mathrm{GeV}$ [28]. The parameters of the neural-network positron finder were modified. To check the acceptance at low $Q^{2}$, which is determined by the positron position in the SRTD, the fiducial region around the impact point of the positron was changed. The resulting systematic uncertainty is typically $\pm 7 \%$ and always smaller than $\pm 25 \%$.

- for the determination of the uncertainties related to the hadronic final state for the $M_{X}^{2}$ method, the effect of the uncertainty in the CAL energy scale $( \pm 2 \%)$ was studied and the parameters in the algorithm that forms EFOs were varied. The non-diffractive slope ( $B$ in Eq. (田) was varied between 1.42 and 1.46. It was checked that a different choice for the functional form of the diffractive contribution in Eq. (4) does not significantly affect the final number of diffractive events. The resulting uncertainty is typically $\pm 8 \%$ and always smaller than $\pm 12 \%$;

- the systematic uncertainties in the measurement of the scattered proton in the LPS were estimated as follows:

- to estimate the sensitivity of the LPS acceptance to the uncertainties in the positions of the beam-line apertures, the lower limits on the distance of closest approach to any of the beam-line elements and to the edge of the sensitive region of each detector were raised from $400 \mu \mathrm{m}$ to $1000 \mu \mathrm{m}$ and from $200 \mu \mathrm{m}$ to $300 \mu \mathrm{m}$, respectively;

- the $x_{L}$ window was varied by \pm 0.01 ;

- the uncertainty in the subtraction of the beam-halo events was estimated by removing the $E+p_{Z}$ cut.

The resulting systematic uncertainty arising from the LPS measurement is typically $\pm 10 \%$ and always smaller than $\pm 25 \%$.

In addition, the $M_{X}, W$ and $t$ dependences in EPSOFT and RAPGAP were varied within the limits allowed by the data, yielding changes in the cross section negligible with respect to all other uncertainties. The relative fraction of vector meson production in EPSOFT was varied by up to $\pm 10 \%$, again with negligible effects on the results.

All the above contributions were summed in quadrature to give the final systematic uncertainties. The normalisation uncertainty due to the luminosity determination is $\pm 1 \%$ for the 1995 data and $\pm 1.5 \%$ for the 1996-97 data and was not included in the sum.

The value of $R^{D}$ was assumed to be zero throughout the analysis. Given the absence of experimental information on $R^{D}$, no attempt was made to quantify the systematic uncertainty entailed by this assumption. 


\section{Cross-section measurements and comparison of the $M_{X}^{2}$ and LPS methods}

The values of $d^{2} \sigma_{\text {diff }}^{\gamma^{*} p} / d M_{X} d t$ and $d \sigma_{\text {diff }}^{\gamma^{*} p} / d M_{X}$ extracted with the LPS method are given in Tables 1 and 2 , respectively. The results obtained with the $M_{X}^{2}$ method are presented in Table 3. All results are corrected to the Born level.

As discussed in Section 8.2, the sample from the $M_{X}^{2}$ method contains a double-dissociative contribution. Since the sample selected with the LPS method has a negligible protondissociative background [28], the contamination in the BPC sample was estimated by directly comparing the results from the two analysis methods.

To achieve this, the ratio, $R_{M_{X}}$, of the average cross section measured with the $M_{X}^{2}$ method and that measured with the LPS method was determined in a single kinematic region corresponding to the bins given in Table 3. The value obtained, $R_{M_{X}}=1.85 \pm 0.38$ (stat.), is attributed to a substantial contribution from the double-dissociative reaction to the cross section measured with the $M_{X}^{2}$ method. In terms of the ratio of the number of doubledissociative events to the total number of events in the sample, $R_{\text {diss }}=\left(1-1 / R_{M_{X}}\right)$, the estimated value of $R_{M_{X}}$ corresponds to $R_{\text {diss }}=(46 \pm 11) \%$. This is consistent with a previous estimate at higher $Q^{2}$ of $(31 \pm 15) \%$ [7].

The results obtained with the $M_{X}^{2}$ method presented in this paper were corrected for the residual double-dissociative background using the measured value of $R_{M_{X}}$. The correction was assumed to be independent of $W$ and $Q^{2}$, in agreement with the hypothesis of vertex factorisation [30]. The values of $d \sigma_{\text {diff }}^{\gamma^{*} p} / d M_{X}$ extracted with the $M_{X}^{2}$ method for each $\left(Q^{2}\right.$, $\left.W, M_{X}\right)$ bin are given in Table 3. The subtraction of the double-dissociative background entails a $\pm 21 \%$ uncertainty in the normalisation of the cross sections obtained with the $M_{X}^{2}$ method.

\section{Results and discussion on the $W$ dependence of the diffractive and total cross sections}

The energy dependence of the photon-dissociative cross sections can be successfully described by a power of $W$, both for photoproduction [3, [] and for DIS [6, [7] - at least in the region of small $x_{I P}$ values where the exchange of subleading Regge trajectories can be neglected. Although the experimental uncertainties are large, the value of this power is different for the two regimes. This is analogous to the behaviour observed for the $W$ dependence of the virtual photon-proton total cross section, $\sigma_{\text {tot }}^{\gamma^{*} p}$ 15, 31: the slow rise of $\sigma_{\text {tot }}^{\gamma^{*} p}$ at high $W$ observed in photoproduction becomes faster at high $Q^{2}$; the transition takes place for $Q^{2} \sim 1 \mathrm{GeV}^{2}$. In this section, the $W$ dependence of the photon-dissociative 
cross section, $d \sigma_{\text {diff }}^{\gamma^{*} p} / d M_{X}$, is studied in this transition region and is compared to the $W$ dependence of $\sigma_{\text {tot }}^{\gamma^{*} p}$ by considering the ratio of $d \sigma_{\text {diff }}^{\gamma^{*} p} / d M_{X}$ to $\sigma_{\text {tot }}^{\gamma^{*} p}$.

\subsection{The $W$ dependence of the diffractive cross section}

Figure 2 shows the values of the diffractive cross sections extracted with the $M_{X}^{2}$ method in the $\mathrm{BPC}$ region as a function of $W$ for three $Q^{2}$ and two $M_{X}$ bins. The form

$$
\frac{d \sigma_{\mathrm{diff}}^{\gamma^{*} p}}{d M_{X}}=A_{i} \cdot W^{a_{\mathrm{diff}}}
$$

was fitted to these data, where $a_{\text {diff }}$ is a global parameter and the normalisation parameters $A_{i}$ were left free to vary for each $\left(Q^{2}, M_{X}\right)$ bin. The results of the fit, taking into account the correlations between adjacent bins, are shown in Fig. 2; they give a good description of the data $\left(\chi^{2} / n d f=0.51\right.$, calculated using the statistical uncertainties only). The fitted value of the power of $W$ is

$$
\left.a_{\text {diff }}=0.510 \pm 0.043 \text { (stat. }\right)_{-0.122}^{+0.102} \text { (syst.). }
$$

Expressing the $W$ dependence of the cross section in terms of an effective Pomeron intercept [1], $\bar{\alpha}_{\mathbb{P}}$, as

$$
\frac{d \sigma_{\text {diff }}^{\gamma^{*} p}}{d M_{X}} \propto\left(W^{2}\right)^{2 \bar{\alpha}_{\mathbb{P}}-2},
$$

the fitted value of $a_{\text {diff }}$ corresponds to

$$
\left.\bar{\alpha}_{I P}=1.128 \pm 0.011 \text { (stat.) }\right)_{-0.030}^{+0.026} \text { (syst.). }
$$

This value of $\bar{\alpha}_{\mathbb{P}}$ can, in turn, be related to the Pomeron intercept, $\alpha_{\mathbb{P}}(0)$, via

$$
\bar{\alpha}_{\mathbb{P}}=\alpha_{\mathbb{P}}(0)-\alpha_{\mathbb{P}}^{\prime} \cdot|\bar{t}|
$$

where $|\bar{t}|$ is the mean value of $|t|$. The value of $\alpha_{\mathbb{P}}(0)$, obtained assuming $\alpha_{\mathbb{P}}^{\prime}=0.25 \mathrm{GeV}^{-2}$ and using $|\bar{t}|=0.13 \mathrm{GeV}^{2}$ [28, 32], is $\alpha_{I P}(0)=1.161 \pm 0.011$ (stat.) ${ }_{-0.030}^{+0.026}$ (syst.); it is shown in Fig. 3 together with the values determined from photoproduction and from higher$Q^{2}$ measurements [3, 4, 6, 7]. The quoted systematic uncertainty does not include the uncertainty on $\alpha_{I P}^{\prime}$, which was also not included in the other results presented in Fig. 3 . The value of $\alpha_{\mathbb{P}}(0)$ from the present measurement at low $Q^{2}$ does not differ significantly from the values at higher $Q^{2}$.

Equation (5) was also fitted to the data allowing different values for the parameter $a_{\text {diff }}$ in the three $Q^{2}$ bins of the measurement; the three resulting values of $a_{\text {diff }}$ are compatible with the global value. The data used in the fit have values of $x_{I P}$ typically much smaller than 0.01 , with the exception of the bin with lowest $W$ and highest $M_{X}$ values, which 
receives contributions from $x_{I P}$ values up to $x_{I P}=0.018$. It was assumed that Pomeron exchange dominates in this region, and no attempt was made to include secondary Reggeon exchange in the fit. Finally, it should be noted that a possible $W$ dependence of the double-dissociative fraction would affect the extracted value of $\alpha_{I P}$.

Figure 3 also shows $\alpha_{\mathbb{P}}(0)$ as obtained from the ALLM97 parameterisation [33] of the $\gamma^{*} p$ total cross section, which gives a good representation of the inclusive $F_{2}$ data for the entire $Q^{2}$ range. The value of $\alpha_{I P}(0)$ from ALLM97 is consistent with the present determination from the diffractive data in the BPC region, whereas in the DIS region it is higher than the $\mathrm{H} 1$ and ZEUS diffractive measurements [6,7].

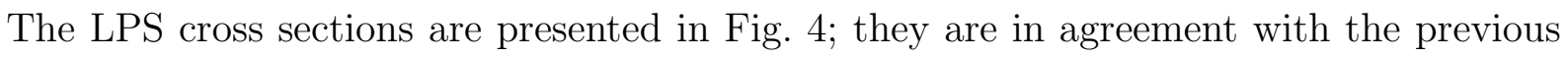
ZEUS measurements at large $Q^{2}$ and with the present BPC data obtained with the $M_{X}^{2}$ method. The previous ZEUS data [7] obtained by the $M_{X}^{2}$ method have also been corrected for the residual double-dissociative background using the value of $R_{M_{X}}$ given in Section 10; to make a direct comparison with the earlier data, the BPC cross sections from Table 3 have been interpolated to $M_{X}=5$ and $11 \mathrm{GeV}$ using bin-centring corrections based on EPSOFT. The solid lines in Fig. 田 correspond to the fit of Eq. (5) to the BPC data alone, which also provides a good description of the DIS data (dashed lines). Figures 3 and 4 thus show that the $W$ dependence of the inclusive diffractive cross section exhibits no significant changes from the BPC to the DIS region.

\subsection{Comparison of the $W$ dependence of the diffractive and the total cross sections}

The $W$ dependences of the diffractive and total cross sections were directly compared by studying their ratio

$$
r=\frac{d \sigma_{\text {diff }}^{\gamma^{*} p} / d M_{X}}{\sigma_{\text {tot }}^{\gamma^{*} p}} .
$$

This ratio is plotted as a function of $W$ in Fig. 5, where the values of the diffractive cross sections shown in Fig. 1 were divided by the corresponding values of the $\gamma^{*} p$ total cross section, $\sigma_{\text {tot }}^{\gamma^{*} p} 15,34$. The lines denote the fit shown in Fig. 1 divided by the corresponding values of the ALLM97 parameterisation for $\sigma_{\text {tot }}^{\gamma^{*} p}$. The lines give a good representation of all the data. While there is a clear increase in $r$ as a function of $W$ for $Q^{2}<1 \mathrm{GeV}^{2}$, for higher $Q^{2}$ the distribution is flat in $W$.

The form $r=N_{i} \cdot W^{\rho}$ was fitted to the BPC data measured with the $M_{X}^{2}$ method; here, $\rho$ is a global parameter and the normalisation parameters, $N_{i}$, were left free to vary for each $\left(Q^{2}, M_{X}\right)$ bin. The fit gives a good description (not shown) of the data with $\rho=0.24 \pm 0.07$, where the uncertainty is derived from the fit, consistent with the expectation [7] from Regge theory that 


$$
r=\frac{\left(d \sigma_{\text {diff }}^{\gamma^{*} p} / d M_{X}\right)}{\sigma_{\text {tot }}^{\gamma^{*} p}} \propto \frac{\left(W^{2}\right)^{2 \bar{\alpha}_{\mathbb{P}}-2}}{\left(W^{2}\right)^{\alpha_{\mathbb{P}}(0)-1}}=W^{2\left(2 \bar{\alpha}_{\mathbb{P}}-\alpha_{\mathbb{P}}(0)-1\right)} \approx W^{0.19}
$$

This result suggests a different behaviour from that found in the DIS region, where the value $\rho=0.00 \pm 0.03$ [7] indicates that the diffractive and inclusive cross sections have the same $W$ dependence, contrary to the expectations of Regge theory.

In summary, in the BPC region the $W$ dependence of the diffractive cross section is compatible with that expected from Regge phenomenology. The ratio between diffractive and total cross sections grows with $W$ at a rate consistent with Regge theory. This is in contrast to the DIS region, where the expectations of Regge theory for the ratio of diffractive and total cross sections are not fulfilled, since the ratio is flat as a function of $W$. This difference in the $W$ dependence of the ratio is reflected in the fact that the values of $\alpha_{I P}(0)$ extracted from the diffractive cross section and from $\sigma_{\text {tot }}^{\gamma^{*} p}$ are similar in the $\mathrm{BPC}$ region, but not in the DIS region.

\section{Results and discussion on the $Q^{2}$ dependence of diffractive and total cross sections}

The $Q^{2}$ dependence of $\sigma_{\text {tot }}^{\gamma^{*} p}$ has been observed to change around $Q^{2} \sim 1 \mathrm{GeV}^{2}$ 《15]: compared to the approximate $1 / Q^{2}$ scaling behaviour found at high $Q^{2}$, data at $Q^{2} \lesssim 1 \mathrm{GeV}^{2}$ exhibit a weaker $Q^{2}$ dependence, with $\sigma_{\text {tot }}^{\gamma^{*} p}$ being nearly independent of $Q^{2}$ at the lowest $Q^{2}$ values measured. This is consistent with the expectation from the conservation of the electromagnetic current that $\sigma_{\text {tot }}^{\gamma^{*} p}$ approaches a constant or, equivalently, that $F_{2}$ vanishes like $Q^{2}$ as $Q^{2} \rightarrow 0$.

In this section, the $Q^{2}$ dependence of the diffractive cross section, $d \sigma_{\text {diff }}^{\gamma^{*} p} / d M_{X}$, is studied and the question is addressed of whether and where a transition similar to that observed for $\sigma_{\text {tot }}^{\gamma^{*} p}$ occurs for the diffractive dissociation of virtual photons.

\subsection{The $Q^{2}$ dependence of the diffractive cross section}

Figure 6 shows the diffractive cross sections, $d \sigma_{\text {diff }}^{\gamma^{*} p} / d M_{X}$, as a function of $Q^{2}$ in bins of $W$ and $M_{X}$. The present measurements are plotted together with previous ZEUS results [7], obtained with the $M_{X}^{2}$ method in the DIS region, and $\mathrm{H1}$ results [35], obtained with the rapidity-gap method in photoproduction for $M_{N}<1.6 \mathrm{GeV}$ and $|t|<1 \mathrm{GeV}^{2}$; bin-centring corrections based on EPSOFT, analogous to those described in Section 11.1, were applied, where necessary, to both ZEUS and H1 results. No further corrections were applied to the H1 data; notably, no attempt was made to correct for the double-dissociative background. 
In Fig. 6, a change in the $Q^{2}$ dependence of $d \sigma_{\text {diff }}^{\gamma^{*} p} / d M_{X}$ as $Q^{2}$ increases is apparent and is similar to that observed in the $\sigma_{\text {tot }}^{\gamma^{*} p}$ data: at low $Q^{2}$, the data do not exhibit a strong $Q^{2}$ dependence, while at larger $Q^{2}$, the cross section falls rapidly for increasing $Q^{2}$. Figure 7 shows $x_{\mathbb{P}} F_{2}^{D(3)}$ as a function of $Q^{2}$ for fixed $W$ and $M_{X}$; while at large $Q^{2}$ the data do not exhibit a strong $Q^{2}$ dependence, $x_{I P} F_{2}^{D(3)}$ falls by a factor of about ten between $Q^{2} \approx 8 \mathrm{GeV}^{2}$ and $Q^{2} \approx 0.2 \mathrm{GeV}^{2}$.

\subsection{Discussion}

The diffractive dissociation of the virtual photon can be described by perturbative QCD (pQCD) since the photon's virtuality, $Q^{2}$, provides a hard scale. In particular, in the proton rest frame, the reaction can be viewed as a sequence of three successive processes 5 , 36]: the photon fluctuates into a $q \bar{q}$ (or $q \bar{q} g$ ) state, the $q \bar{q}$ dipole scatters off the proton target and, finally, the scattered $q \bar{q}$ pair produces the final state. At high centre-of-mass energies of the $\gamma p$ system, these processes are widely separated in time. The $q \bar{q}$ fluctuation is described in terms of the photon wave-function derived from QCD. The interaction of the $q \bar{q}$ dipole with the proton is mediated, in lowest order, by the exchange of two gluons in a colour-singlet state.

The present results have been compared to the model of Bartels et al. (BEKW) [37]. In this model, neglecting the contribution of longitudinally polarised photons, the dominant (leading-twist) contributions to the diffractive structure function in the kinematic domain of the present measurement come from the fluctuations of the photon into either a $q \bar{q}$ pair $\left(F_{q \bar{q}}^{T}\right)$ or a $q \bar{q} g$ state $\left(F_{q \bar{q} g}^{T}\right)$. The $\beta$ spectra of these two components are determined by rather general properties of the photon wave-function: $F_{q \bar{q}}^{T}$ behaves like $\beta(1-\beta)$ and $F_{q \bar{q} g}^{T}$ like $(1-\beta)^{\gamma}$, where $\gamma=3.9[7,37]$. At large $\beta, q \bar{q}$ production dominates over $q \bar{q} g$ production, while, at small $\beta, q \bar{q} g$ production becomes dominant. $F_{q \bar{q}}^{T}$ has no $Q^{2}$ dependence; $F_{q \bar{q} g}^{T}$ is of order $\alpha_{S}$ and has a logarithmic $Q^{2}$ dependence of the type $\log \left(1+Q^{2} / Q_{0}^{2}\right)$, where the scale parameter $Q_{0}^{2}$ is taken to be $1 \mathrm{GeV}^{2}$. The model does not fix the $x_{\mathbb{P}}$ dependence of $F_{q \bar{q}}^{T}$ and $F_{q \bar{q} g}^{T}$, but assumes for both a power-like behaviour $x_{\mathbb{P}}^{-n_{\text {diff }}\left(Q^{2}\right)}$, where the exponent $n_{\text {diff }}$ is determined from fits to the data.

A comparison of the BEKW parameterisation with the present data is shown in Figs. 6 and 6 . The values of the parameters, including the normalisation of the $F_{q \bar{q}}^{T}$ and $F_{q \bar{q} g}^{T}$ components, were taken from a fit to the previous ZEUS results [7], with the exception of the $x_{\mathbb{P}}$ exponent, for which a constant value corresponding to $a_{\text {diff }} / 2$, determined from Eq. (5), was used. The DIS data at high $Q^{2}$ constrain the parameterisation of the $\beta$ dependence of $F_{q \bar{q}}^{T}$ (dashed lines) at low $M_{X}$ and of $F_{q \bar{q} g}^{T}$ (dotted lines) at high $M_{X}$. The logarithmic $Q^{2}$ dependence of $F_{q \bar{q} g}^{T}$ is probed only in the highest- $Q^{2}$ region and is less well constrained.

The $Q^{2}$ dependence of $F_{q \bar{q} g}^{T}$ becomes crucial in the transition to low $Q^{2}$. In fact, as $Q^{2}$ decreases from the DIS into the BPC region, for a given value of $M_{X}, \beta$ also decreases: 
the BPC data in Figs. 6 and 7 correspond to values of $\beta$ that are typically two orders of magnitude smaller than those in the DIS data and thus, in the BPC region, the contribution from the fluctuation of the photon into a $q \bar{q} g$ system becomes dominant. While extrapolating the BEKW parameterisation to low $Q^{2}$ may not be formally justified, it is interesting to note that, in $F_{q \bar{q} g}^{T}$, conservation of the electromagnetic current is assured by the fact that $\log \left(1+Q^{2} / Q_{0}^{2}\right)$ vanishes like $Q^{2} / Q_{0}^{2}$ as $Q^{2} \rightarrow 0$. The transition from the linear behaviour at low $Q^{2}$ to the logarithmic behaviour at higher $Q^{2}$ is controlled by the scale parameter $Q_{0}^{2}$; the choice $Q_{0}^{2}=1 \mathrm{GeV}^{2}$ successfully describes the BPC data.

\subsection{Comparison of the $Q^{2}$ dependence of the diffractive and the total cross sections}

Figure 8 shows the ratio $r=\left(d \sigma_{\text {diff }}^{\gamma^{*} p} / d M_{X}\right) / \sigma_{\text {tot }}^{\gamma^{*} p}$ as a function of $Q^{2}$ for different $W$ and $M_{X}$ bins. At low $Q^{2}$, the $Q^{2}$ dependence of the diffractive cross section is similar to that of $\sigma_{\text {tot }}^{\gamma^{*} p}$. In the DIS regime, $d \sigma_{\text {diff }}^{\gamma^{*} p} / d M_{X}$ decreases with $Q^{2}$ more rapidly than $\sigma_{\text {tot }}^{\gamma^{*} p}$. This is more evident for small values of $M_{X}$. In addition, the ratio $r$ appears to increase between the $\mathrm{BPC}$ and the DIS regions.

Also shown in the figure are the results of the BEKW parameterisation of the diffractive cross-section $d \sigma_{\text {diff }}^{\gamma^{*} p} / d M_{X}$, shown in Fig. 6, divided by the values of $\sigma_{\text {tot }}^{\gamma^{*} p}$ given by the ALLM97 parameterisation [33. There is reasonable agreement between these parameterisations and the data, indicating that the data may be qualitatively described by an appropriate choice of the relative fractions of the $q \bar{q}$ and $q \bar{q} g$ contributions.

\section{Summary}

The diffractive dissociation of virtual photons, $\gamma^{*} p \rightarrow X p$, has been studied at HERA at low $Q^{2}\left(0.17<Q^{2}<0.70 \mathrm{GeV}^{2}\right)$ and in deep inelastic scattering (DIS) $\left(3<Q^{2}<\right.$ $80 \mathrm{GeV}^{2}$ ). The diffractive signal has been selected either by requiring the detection of a final-state proton with at least $97 \%$ of the incoming proton-beam energy, or by exploiting the different properties of the $M_{X}$ distributions for diffractive and non-diffractive events.

The $W$ dependence of the low- $Q^{2}$ cross-section data obtained with the $M_{X}^{2}$ method $(3<$ $M_{X}<12.2 \mathrm{GeV}$ ) has been found to be compatible with a single power of $W$, which corresponds to a Pomeron intercept of $\alpha_{I P}(0)=1.161 \pm 0.011$ (stat. $)_{-0.030}^{+0.026}$ (syst.). This is consistent with that previously observed in the DIS regime. Thus, the significant change in the $W$ dependence exhibited by the $\gamma^{*} p$ total cross section in the transition from low $Q^{2}$ to DIS is not visible in the diffractive cross section. To elucidate this difference, the $W$ dependence of the ratio, $r$, of the diffractive cross section to the $\gamma^{*} p$ total cross section was studied at low $Q^{2}$ and was found to rise with $W, r \propto W^{0.24 \pm 0.07}$, in agreement with 
the expectation from Regge theory. This is in contrast to the observation at higher $Q^{2}$ that this ratio is independent of $W$.

The $Q^{2}$ dependence of the diffractive cross section changes as $Q^{2}$ increases up to the DIS regime: while at low $Q^{2}$ the data do not exhibit a strong $Q^{2}$ dependence, at larger $Q^{2}$ the cross section falls rapidly for increasing $Q^{2}$. This change of behaviour occurs for values of $Q^{2}$ around $1 \mathrm{GeV}^{2}$ and is analogous to that observed in the total $\gamma^{*} p$ cross section. The ratio of the diffractive cross section to the $\gamma^{*} p$ total cross section was studied as a function of $Q^{2}$. At low $Q^{2}$, the ratio $r$ shows little dependence on $Q^{2}$, indicating that the $Q^{2}$ dependence of the diffractive cross section is similar to that of $\sigma_{\text {tot }}^{\gamma^{*} p}$. The ratio increases between the BPC and the DIS regions. In the DIS regime for low $M_{X}$, the ratio decreases with increasing $Q^{2}$, indicating that the diffractive cross section decreases with $Q^{2}$ more rapidly than the $\gamma^{*} p$ total cross section.

The main features of the data, reproduced by a parameterisation based on the BEKW model, indicate that the framework in which the incoming virtual photon fluctuates into a quark-antiquark pair is, in general, adequate to describe diffractive processes in ep collisions from the BPC to the DIS region. At the same time, the data suggest the increasing importance of the contribution from $q \bar{q} g$ states at low $Q^{2}$. It is interesting that the ratio of the diffractive cross section to the total cross section shows a change from a $W^{0.24 \pm 0.07}$ dependence for $Q^{2}<0.7 \mathrm{GeV}^{2}$ to $W^{0.00 \pm 0.03}$ for $Q^{2}>3 \mathrm{GeV}^{2}$. This complex behaviour of diffraction as a function of both $Q^{2}$ and $W$ reveals a rich testing ground for future theoretical models.

\section{Acknowledgements}

We thank the DESY directorate for their encouragement, and acknowledge the support of the DESY computing and network services. We are especially grateful to the HERA machine group: collaboration with them was crucial to the successful installation and operation of the leading proton spectrometer. The design, construction and installation

of the ZEUS detector have been made possible by the ingenuity and efforts of many people from DESY and home institutes who are not listed as authors. Finally, it is a pleasure to thank J. Bartels, K. Golec-Biernat, N.N. Nikolaev, M.G. Ryskin and M. Strikman for many useful discussions.

\section{References}

[1] P.D.B. Collins, An Introduction to Regge Theory and High Energy Physics, Cambridge University Press, Cambridge (1977). 
[2] A. Donnachie and P.V. Landshoff, Nucl. Phys. B244, 322 (1984);

A. Donnachie and P.V. Landshoff, Phys. Lett. B296, 227 (1992);

see also:

J.R. Cudell, K. Kang and S.K. Kim, Phys. Lett. B395, 311 (1997);

Particle Data Group, D.E. Groom et al., Eur. Phys. J. C15, 1 (2000).

[3] H1 Collab., C. Adloff et al., Z. Phys. C74, 221 (1997).

[4] ZEUS Collab., J. Breitweg et al., Z. Phys. C75, 421 (1997).

[5] See, for example, H. Abramowicz, Int. J. Mod. Phys. A15S1, 495 (2000) and references therein.

[6] H1 Collab., C. Adloff et al., Z. Phys. C76, 613 (1997).

[7] ZEUS Collab., J. Breitweg et al., Eur. Phys. J. C6, 43 (1999).

[8] L.N. Hand, Phys. Rev. 129, 1834 (1963).

[9] G. Ingelman and K. Janson-Prytz, Proceedings of the Workshop "Physics at HERA", Volume 1, W. Buchmüller, G. Ingelman (eds.), DESY (1992), p. 233;

G. Ingelman and K. Prytz, Z. Phys. C58, 285 (1993).

[10] M.F. McDermott and G. Briskin, Proceedings of the Workshop "Future Physics at HERA", Volume 2, G.Ingelman, A. De Roeck and R. Klanner (eds.), DESY (1996), p. 691, and references therein.

[11] ZEUS Collab., U. Holm (ed.), The ZEUS Detector, Status Report, (unpublished), DESY (1993);

http://www-zeus.desy.de/bluebook/bluebook.html.

[12] N. Harnew et al., Nucl. Instr. and Meth. A279, 290 (1989);

B. Foster et al., Nucl. Phys. Proc. Suppl. B32, 181 (1993);

B. Foster et al., Nucl. Inst. and Meth. A338, 254 (1994).

[13] M. Derrick et al., Nucl. Instr. and Meth. A309,77 (1991);

A. Andresen et al., Nucl. Instr. and Meth. A309, 101 (1991);

A. Caldwell et al., Nucl. Instr. and Meth. A321, 356 (1992);

A. Bernstein et al., Nucl. Instr. and Meth. A336, 23 (1993).

[14] ZEUS Collab., J. Breitweg et al., Phys. Lett. B407, 432 (1997).

[15] ZEUS Collab., J. Breitweg et al., Phys. Lett. B487, 53 (2000).

[16] A. Bamberger et al., Nucl. Instr. and Meth. A382, 419 (1996).

[17] ZEUS Collab., M. Derrick et al., Z. Phys. C73, 253 (1997). 
[18] S. Bentvelsen, J. Engelen and P. Kooijman, in Proceedings of the Workshop on Physics at HERA, Oct. 1991, Volume 1, W. Buchmüller and G. Ingelman (eds.), DESY (1992), p. 23;

K.C. Höger, ibid., p. 43.

[19] G. Briskin, PhD Thesis, Tel Aviv University (1998), DESY-Thesis-1998-036.

[20] F. Jacquet and A. Blondel, Proceedings of the Workshop "Study for an ep Facility for Europe", U. Amaldi (ed.), DESY 79-048 (1979), p. 391.

[21] M. Kasprzak, PhD Thesis, Warsaw University (1996), DESY F35D-96-16.

[22] M. Inuzuka, PhD Thesis, University of Tokyo (1999), KEK Report 99-9.

[23] K. Kwiatkowski, H. Spiesberger and H.-J. Möhring, Comput. Phys. Commun. 69, 155 (1992).

[24] H. Jung, DESY Report 93-182 (1993).

[25] G. Ingelman and P. Schlein, Phys. Lett. B152, 256 (1985).

[26] GEANT 3.13, R. Brun et al., CERN DD/EE/84-1 (1987).

[27] H. Abramowicz, A. Caldwell and R. Sinkus, Nucl. Instr. and Meth. A365, 508 (1995).

[28] ZEUS Collab., J. Breitweg et al., Eur. Phys. J. C1, 81 (1998).

[29] A. Hoecker and V. Kartvelishvili, Nucl. Instr. and Meth. A372, 469 (1996).

[30] See e.g.:

U. Amaldi, M. Jacob and G. Matthiae, Ann. Rev. Nucl. Sci. 26, 385 (1976);

G. Cohen-Tannoudji, D. Levy and M. Souza, Nucl. Phys. B129, 286 (1977);

G. Alberi and G. Goggi, Phys. Rep. 74, 1 (1981);

K. Goulianos, Phys. Rep. 101, 169 (1983);

M. Kamran, Phys. Rep. 108, 275 (1984);

N.P. Zotov and V.A. Tsarev, Sov. Phys. Uspekhi 31, 119 (1988);

G. Giacomelli, Int. J. Mod. Phys. A5, 223 (1990).

[31] ZEUS Collab., J.Breitweg et al., Eur. Phys. J. C7, 609 (1999).

[32] ZEUS Collab., J. Breitweg et al., Eur. Phys. J. C2, 237 (1998).

[33] H.Abramowicz and A.Levy, DESY Report 97-251 (1997).

[34] ZEUS Collab., S. Chekanov et al., Eur. Phys. J. C21, 443 (2001).

[35] H1 Collab., C. Adloff et al., Z.Phys. C74, 221 (1997). 
[36] M. Wüsthoff and A. D. Martin, J. Phys. G25, R309 (1999);

A. Hebecker, Phys. Rep. 331, 1 (2000); Acta Phys. Polon. B30 (1999) 3777;

K. Golec-Biernat and M. Wüsthoff, Eur. Phys. J. C20, 313 (2001).

[37] J. Bartels et al., Eur. Phys. J. C7, 443 (1999). 


\begin{tabular}{|c|c|c||c|c|c||c|c|c||c|}
\hline$Q_{\min }^{2}$ & $Q_{\max }^{2}$ & $Q^{2}$ & $W_{\min }$ & $W_{\max }$ & $W$ & $M_{X, \min }$ & $M_{X, \max }$ & $M_{X}$ & $\frac{d^{2} \sigma_{\text {dif }}^{\gamma^{*} p}}{d M_{X} d t}$ \\
\hline \multicolumn{3}{|c||}{$\left(\mathrm{GeV}^{2}\right)$} & \multicolumn{3}{c||}{$(\mathrm{GeV})$} & \multicolumn{3}{|c||}{$(\mathrm{GeV})$} & $\left(\mu \mathrm{b} / \mathrm{GeV}^{3}\right)$ \\
\hline \hline 0.17 & 0.70 & \multirow{2}{*}{0.39} & 90 & 250 & 130 & 3.00 & 6.05 & 5 & $0.867 \pm 0.186_{-0.139}^{+0.170}$ \\
& & & 90 & 250 & 130 & 6.05 & 12.20 & 11 & $0.144 \pm 0.043_{-0.028}^{+0.040}$ \\
& & & 165 & 250 & 210 & 12.20 & 38.00 & 22 & $0.202 \pm 0.074_{-0.052}^{+0.034}$ \\
\hline \hline 3 & \multirow{2}{*}{9} & \multirow{2}{*}{4} & 80 & 165 & 130 & 3.00 & 6.05 & 5 & $0.346 \pm 0.063_{-0.039}^{+0.081}$ \\
& & & 165 & 250 & 210 & 3.00 & 6.05 & 5 & $0.349 \pm 0.080_{-0.095}^{+0.083}$ \\
& & & 80 & 165 & 130 & 6.05 & 12.20 & 11 & $0.172 \pm 0.036_{-0.035}^{+0.019}$ \\
& & & 165 & 250 & 210 & 6.05 & 12.20 & 11 & $0.350 \pm 0.115_{-0.133}^{+0.050}$ \\
& & & 165 & 250 & 210 & 12.20 & 33.00 & 22 & $0.098 \pm 0.024_{-0.025}^{+0.006}$ \\
\hline \hline \multirow{2}{*}{9} & \multirow{2}{*}{80} & 27 & 80 & 165 & 130 & 3.00 & 6.05 & 5 & $0.042 \pm 0.008_{-0.005}^{+0.008}$ \\
& & & 165 & 250 & 210 & 3.00 & 6.05 & 5 & $0.044 \pm 0.012_{-0.007}^{+0.008}$ \\
& & & 80 & 165 & 130 & 6.05 & 12.20 & 11 & $0.038 \pm 0.006_{-0.005}^{+0.002}$ \\
& & & 165 & 250 & 210 & 6.05 & 12.20 & 11 & $0.029 \pm 0.007_{-0.008}^{+0.003}$ \\
& & & 165 & 250 & 210 & 12.20 & 33.00 & 22 & $0.014 \pm 0.003_{-0.002}^{+0.002}$ \\
\hline
\end{tabular}

Table 1: The values of $d^{2} \sigma_{\text {diff }}^{\gamma^{*} p} / d M_{X} d t$ measured with the LPS method in the range $0.073<|t|<0.40 \mathrm{GeV}^{2}$ with the bin ranges indicated. The data are at $\langle t\rangle=0.17 \mathrm{GeV}^{2}$. The first and second error values represent the statistical and systematic uncertainties, respectively. The normalisation uncertainty related to the luminosity measurement is not included in the systematic uncertainty. 


\begin{tabular}{|c|c|c||c|c|c||c|c|c||c|}
\hline$Q_{\min }^{2}$ & $Q_{\max }^{2}$ & $Q^{2}$ & $W_{\min }$ & $W_{\max }$ & $W$ & $M_{X, \min }$ & $M_{X, \max }$ & $M_{X}$ & $\frac{d \sigma_{\text {diff }}^{*^{*} p}}{d M_{X}}$ \\
\hline \multicolumn{3}{|c||}{$\left(\mathrm{GeV}^{2}\right)$} & \multicolumn{3}{|c||}{$(\mathrm{GeV})$} & \multicolumn{3}{c||}{$(\mathrm{GeV})$} & $(\mu \mathrm{b} / \mathrm{GeV})$ \\
\hline \hline \multirow{2}{*}{0.17} & \multirow{2}{*}{0.70} & \multirow{2}{*}{0.39} & 90 & 250 & 130 & 3.00 & 6.05 & 5 & $0.511 \pm 0.110_{-0.082}^{+0.100}$ \\
& & & 90 & 250 & 130 & 6.05 & 12.20 & 11 & $0.086 \pm 0.026_{-0.017}^{+0.024}$ \\
& & & 165 & 250 & 210 & 12.20 & 38.00 & 22 & $0.120 \pm 0.044_{-0.031}^{+0.020}$ \\
\hline \hline \multirow{2}{*}{3} & \multirow{2}{*}{9} & \multirow{2}{*}{4} & 80 & 165 & 130 & 3.00 & 6.05 & 5 & $0.172 \pm 0.031_{-0.019}^{+0.040}$ \\
& & & 165 & 250 & 210 & 3.00 & 6.05 & 5 & $0.175 \pm 0.040_{-0.047}^{+0.042}$ \\
& & & 80 & 165 & 130 & 6.05 & 12.20 & 11 & $0.084 \pm 0.017_{-0.017}^{+0.009}$ \\
& & & 165 & 250 & 210 & 6.05 & 12.20 & 11 & $0.174 \pm 0.057_{-0.066}^{+0.025}$ \\
& & & 165 & 250 & 210 & 12.20 & 33.00 & 22 & $0.055 \pm 0.014_{-0.014}^{+0.003}$ \\
\hline \hline \multirow{2}{*}{9} & \multirow{2}{*}{80} & \multirow{2}{*}{27} & 80 & 165 & 130 & 3.00 & 6.05 & 5 & $0.020 \pm 0.004_{-0.002}^{+0.004}$ \\
& & & 165 & 250 & 210 & 3.00 & 6.05 & 5 & $0.022 \pm 0.006_{-0.004}^{+0.004}$ \\
& & & 80 & 165 & 130 & 6.05 & 12.20 & 11 & $0.019 \pm 0.003_{-0.003}^{+0.001}$ \\
& & & 165 & 250 & 210 & 6.05 & 12.20 & 11 & $0.014 \pm 0.004_{-0.004}^{+0.002}$ \\
& & & 165 & 250 & 210 & 12.20 & 33.00 & 22 & $0.007 \pm 0.001_{-0.001}^{+0.001}$ \\
\hline
\end{tabular}

Table 2: $\quad$ The values of $d \sigma_{\mathrm{diff}}^{\gamma^{*} p} / d M_{X}$ measured with the LPS method with the bin ranges indicated. The first and second error values represent the statistical and systematic uncertainties, respectively. The normalisation uncertainty related to the luminosity measurement is not included in the systematic uncertainty. 


\begin{tabular}{|c|c|c|c|c|c|c|c|c|c|}
\hline$Q_{\min }^{2}$ & $Q_{\max }^{2}$ & $Q^{2}$ & $W_{\min }$ & $W_{\max }$ & $W$ & $M_{X, \min }$ & $M_{X, \max }$ & $M_{X}$ & $\frac{d \sigma_{\text {diff }}^{\gamma^{*} p}}{d M_{X}}$ \\
\hline \multicolumn{3}{|c|}{$\left(\mathrm{GeV}^{2}\right)$} & \multicolumn{3}{|c|}{$(\mathrm{GeV})$} & \multicolumn{3}{|c|}{$(\mathrm{GeV})$} & $(\mu \mathrm{b} / \mathrm{GeV})$ \\
\hline \multirow[t]{5}{*}{0.220} & 0.324 & 0.27 & 90 & 120 & 104 & \multirow[t]{5}{*}{3.00} & \multirow[t]{5}{*}{6.05} & \multirow[t]{5}{*}{4.26} & $0.490 \pm 0.022_{-0.036}^{+0.065}$ \\
\hline & & & 120 & 150 & 134 & & & & $0.557 \pm 0.025_{-0.036}^{+0.056}$ \\
\hline & & & 150 & 180 & 164 & & & & $0.612 \pm 0.029_{-0.027}^{+0.068}$ \\
\hline & & & 180 & 200 & 190 & & & & $0.698 \pm 0.040_{-0.028}^{+0.057}$ \\
\hline & & & 200 & 220 & 210 & & & & $0.768 \pm 0.047_{-0.053}^{+0.080}$ \\
\hline \multirow[t]{5}{*}{0.220} & 0.324 & 0.27 & 90 & 120 & 104 & \multirow[t]{5}{*}{6.05} & \multirow[t]{5}{*}{12.20} & \multirow[t]{5}{*}{8.58} & $0.200 \pm 0.010_{-0.019}^{+0.039}$ \\
\hline & & & 120 & 150 & 134 & & & & $0.218 \pm 0.010_{-0.017}^{+0.033}$ \\
\hline & & & 150 & 180 & 164 & & & & $0.246 \pm 0.012_{-0.010}^{+0.024}$ \\
\hline & & & 180 & 200 & 190 & & & & $0.259 \pm 0.015_{-0.013}^{+0.015}$ \\
\hline & & & 200 & 220 & 210 & & & & $0.291 \pm 0.019_{-0.022}^{+0.025}$ \\
\hline \multirow[t]{4}{*}{0.324} & 0.476 & 0.39 & 90 & 120 & 104 & \multirow[t]{4}{*}{3.00} & \multirow[t]{4}{*}{6.05} & \multirow[t]{4}{*}{4.26} & $0.433 \pm 0.019_{-0.019}^{+0.034}$ \\
\hline & & & 120 & 150 & 134 & & & & $0.455 \pm 0.021_{-0.032}^{+0.039}$ \\
\hline & & & 150 & 180 & 164 & & & & $0.531 \pm 0.027_{-0.034}^{+0.050}$ \\
\hline & & & 180 & 200 & 190 & & & & $0.599 \pm 0.037_{-0.038}^{+0.047}$ \\
\hline \multirow[t]{4}{*}{0.324} & 0.476 & 0.39 & 90 & 120 & 104 & \multirow[t]{4}{*}{6.05} & \multirow[t]{4}{*}{12.20} & \multirow[t]{4}{*}{8.58} & $0.171 \pm 0.008_{-0.008}^{+0.020}$ \\
\hline & & & 120 & 150 & 134 & & & & $0.186 \pm 0.009_{-0.012}^{+0.019}$ \\
\hline & & & 150 & 180 & 164 & & & & $0.200 \pm 0.010_{-0.010}^{+0.016}$ \\
\hline & & & 180 & 200 & 190 & & & & $0.238 \pm 0.015_{-0.011}^{+0.013}$ \\
\hline \multirow[t]{3}{*}{0.476} & 0.700 & 0.58 & 90 & 120 & 104 & \multirow[t]{3}{*}{3.00} & \multirow[t]{3}{*}{6.05} & \multirow[t]{3}{*}{4.26} & $0.373 \pm 0.019_{-0.018}^{+0.031}$ \\
\hline & & & 120 & 150 & 134 & & & & $0.411 \pm 0.022_{-0.033}^{+0.038}$ \\
\hline & & & 150 & 180 & 164 & & & & $0.432 \pm 0.026_{-0.036}^{+0.048}$ \\
\hline \multirow[t]{3}{*}{0.476} & 0.700 & 0.58 & 90 & 120 & 104 & \multirow[t]{3}{*}{6.05} & \multirow[t]{3}{*}{12.20} & \multirow[t]{3}{*}{8.58} & $0.149 \pm 0.008_{-0.011}^{+0.017}$ \\
\hline & & & 120 & 150 & 134 & & & & $0.166 \pm 0.009_{-0.012}^{+0.014}$ \\
\hline & & & 150 & 180 & 164 & & & & $0.162 \pm 0.010_{-0.010}^{+0.010}$ \\
\hline
\end{tabular}

Table 3: The diffractive cross-sections $d \sigma_{\text {diff }}^{\gamma^{*} p} / d M_{X}$ measured with the $M_{X}^{2}$ method with the bin ranges indicated. The first and second error values represent the statistical and systematic uncertainties, respectively. The $\pm 21 \%$ systematic uncertainty due to the double-dissociation correction is not included in the systematic uncertainty, nor is the normalisation uncertainty related to the luminosity measurement. 

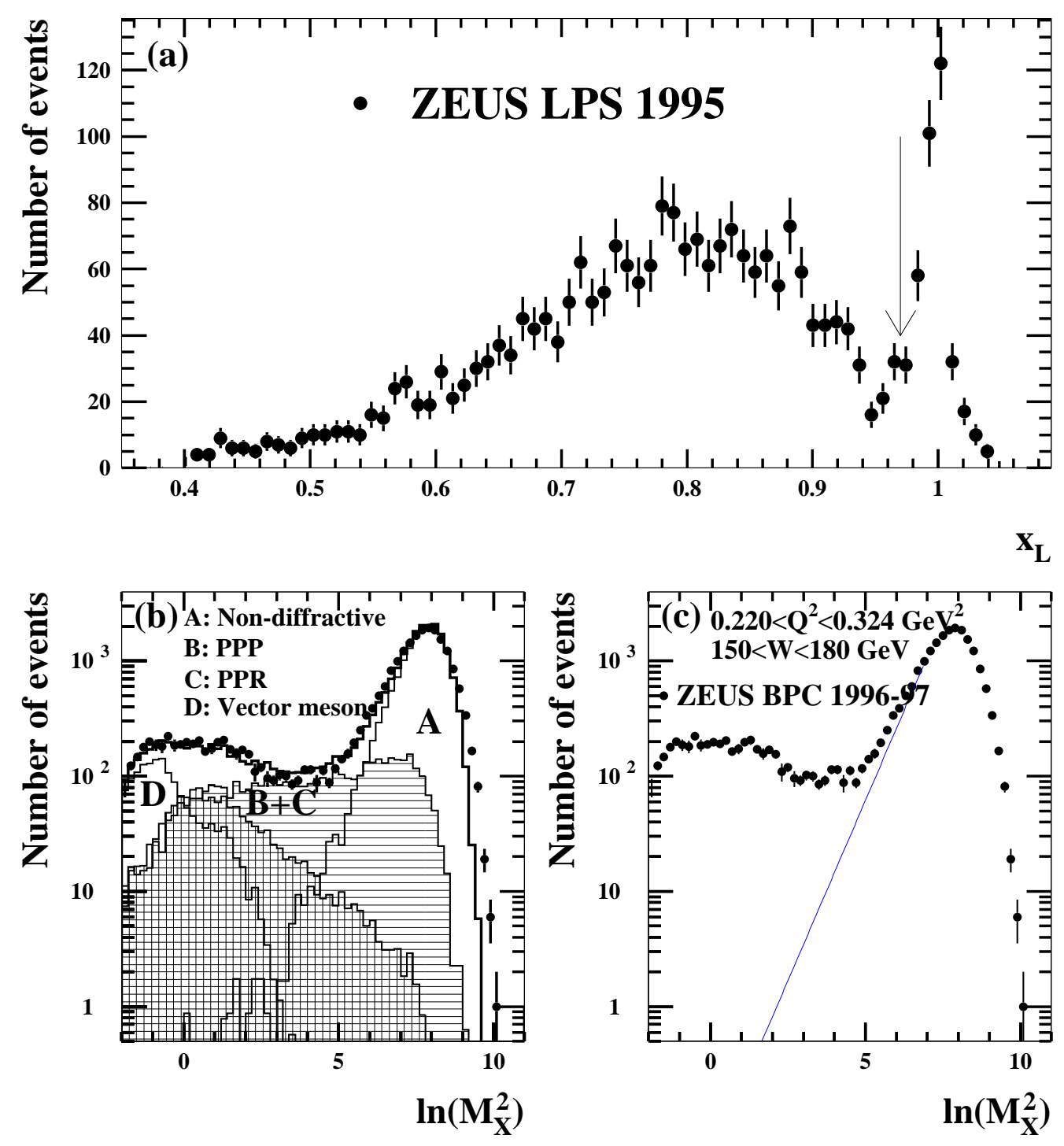

Figure 1: (a) The $x_{L}$ spectrum as measured in BPC events with the LPS; (b) and (c) the $\ln M_{X}^{2}$ distribution $\left(M_{X}\right.$ in $\mathrm{GeV}$ ) of the BPC data in the region $0.220<Q^{2}<0.324 \mathrm{GeV}^{2}$ and $150<W<180 \mathrm{GeV}$. In (a), the position of the arrow indicates the value $x_{L}=0.97$ used in the selection. In (b), the data are compared to the mixture of four kinds of EPSOFT MC events described in the text: region $A$ corresponds to non-diffractive events, $B+C$ to the sum of the IPIPIP and IPIPIR contributions and D to the vector-meson contribution. In (c), the straight line shows the exponential slope, resulting from the fit described in the text, for non-diffractive events. 


\section{ZEUS}
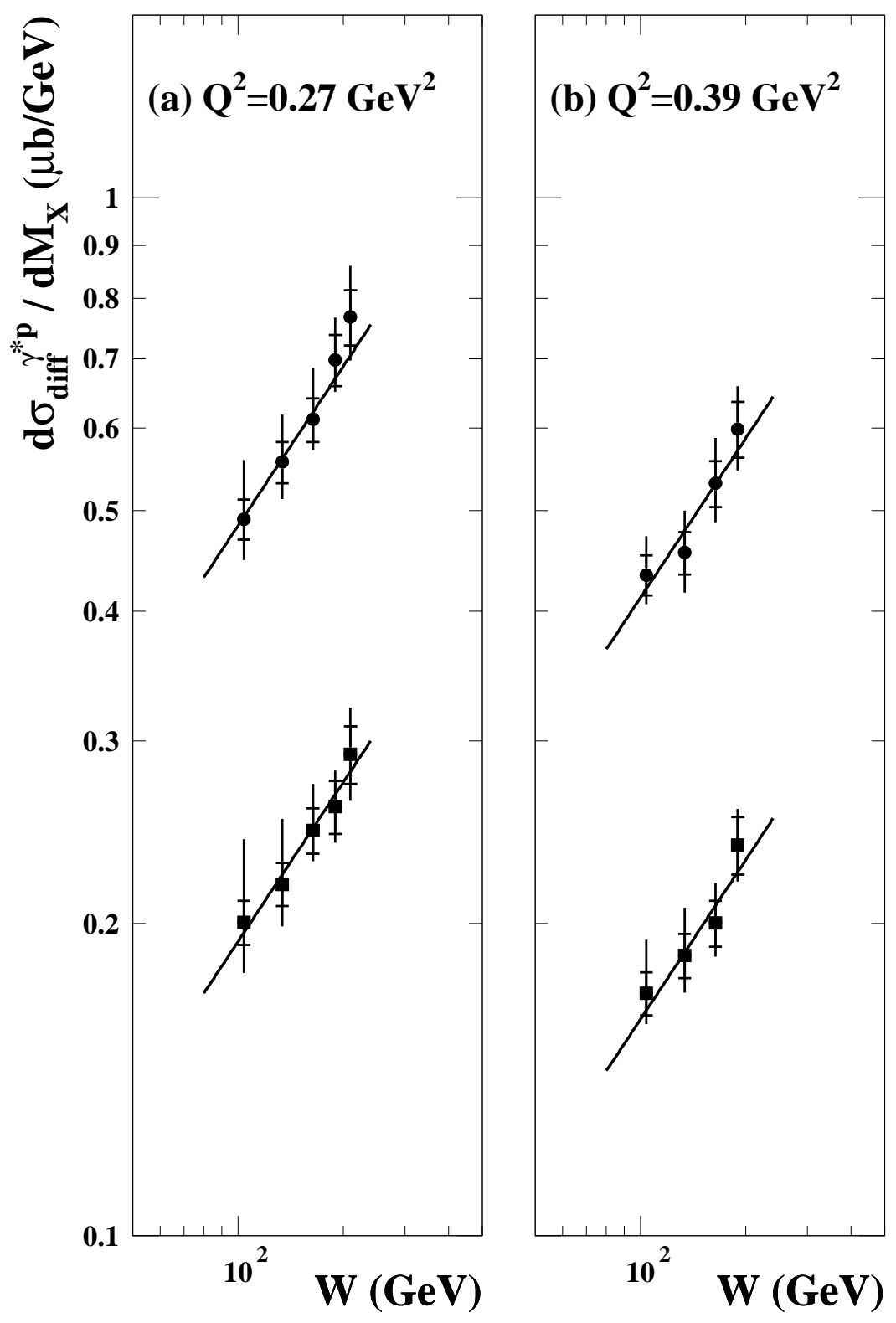

(c) $\mathrm{Q}^{2}=0.58 \mathrm{GeV}^{2}$

ZEUS BPC 1996-97

- $\mathrm{M}_{\mathrm{X}}=4.26 \mathrm{GeV}$

- $\mathrm{M}_{\mathrm{X}}=8.58 \mathrm{GeV}$

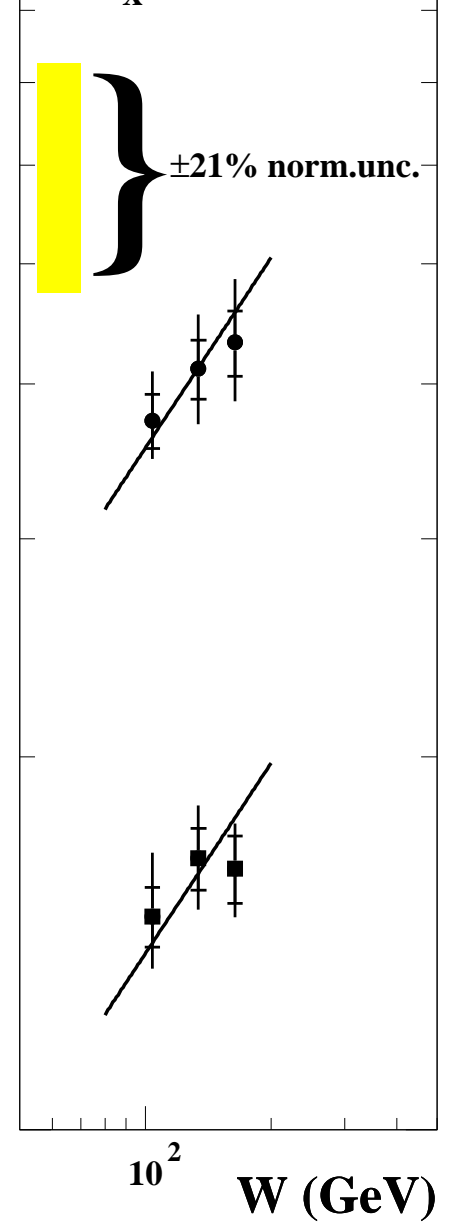

Figure 2: Diffractive cross sections for (a) $Q^{2}=0.27 \mathrm{GeV}^{2}$, (b) $Q^{2}=0.39 \mathrm{GeV}^{2}$, and (c) $Q^{2}=0.58 \mathrm{GeV}^{2}$ for two different $M_{X}$ ranges as a function of $W$. The inner bars indicate the size of the statistical uncertainties; the outer bars show the size of the statistical and systematic uncertainties added in quadrature. The points were corrected for the double-dissociative background; the associated $\pm 21 \%$ normalisation uncertainty is not included, but is shown separately as a shaded error band. The normalisation uncertainty associated with the luminosity measurement is not shown. The lines show the results of the fit described in the text. 


\section{ZEUS}

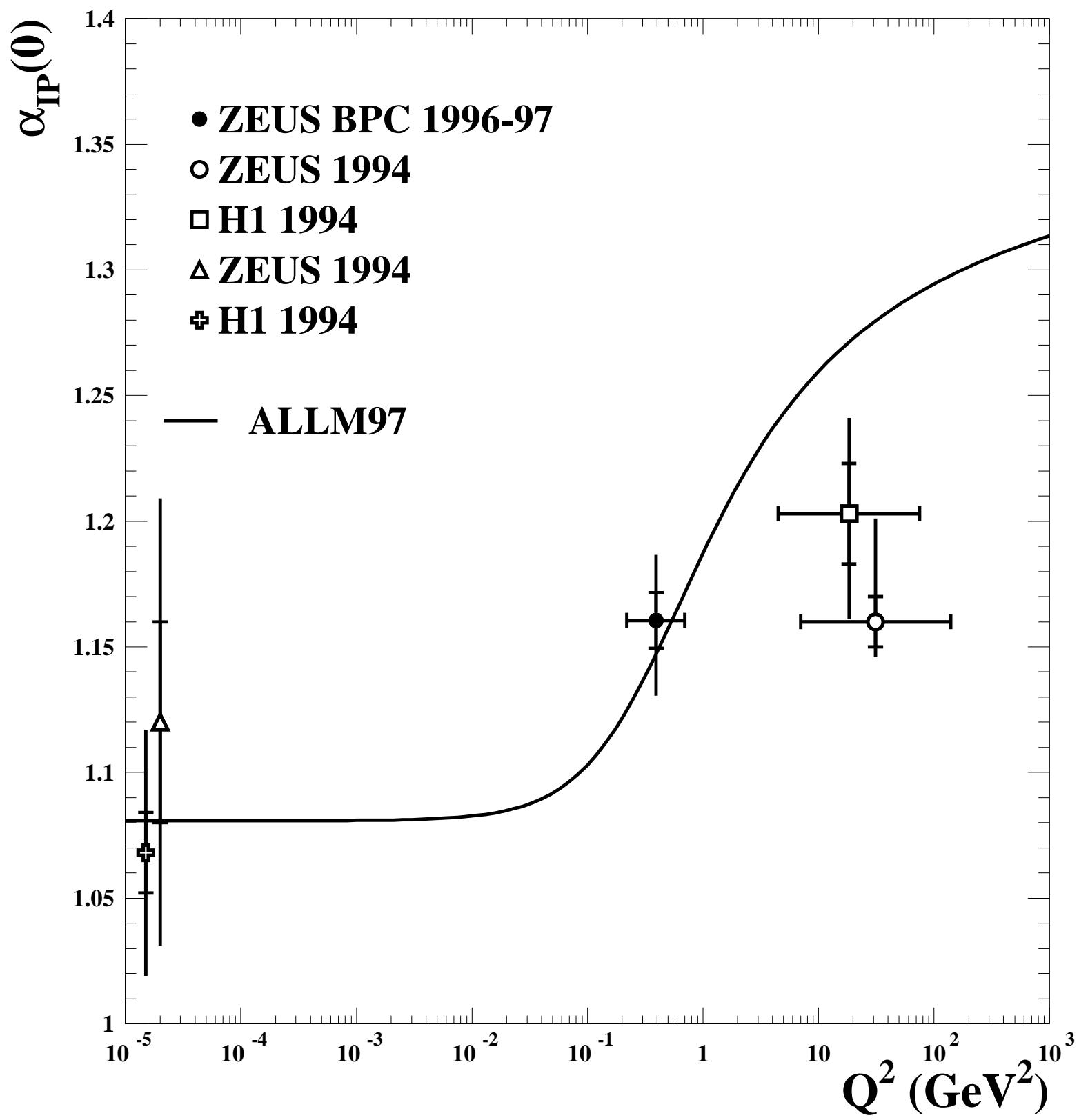

Figure 3: Results for $\alpha_{\mathbb{P}}(0)$ in different $Q^{2}$ regions. The value of $\alpha_{\mathbb{P}}(0)$ obtained from this analysis is shown as the solid circle. The open symbols show the results from the photoproduction [3, [7] and DIS diffractive analyses [9, [7]. The inner bars indicate the size of the statistical uncertainties; the outer bars show the size of the statistical and systematic uncertainties added in quadrature. The line is from the ALLM97 parameterisation [3] of the $\gamma^{*} p$ total cross-section data. 


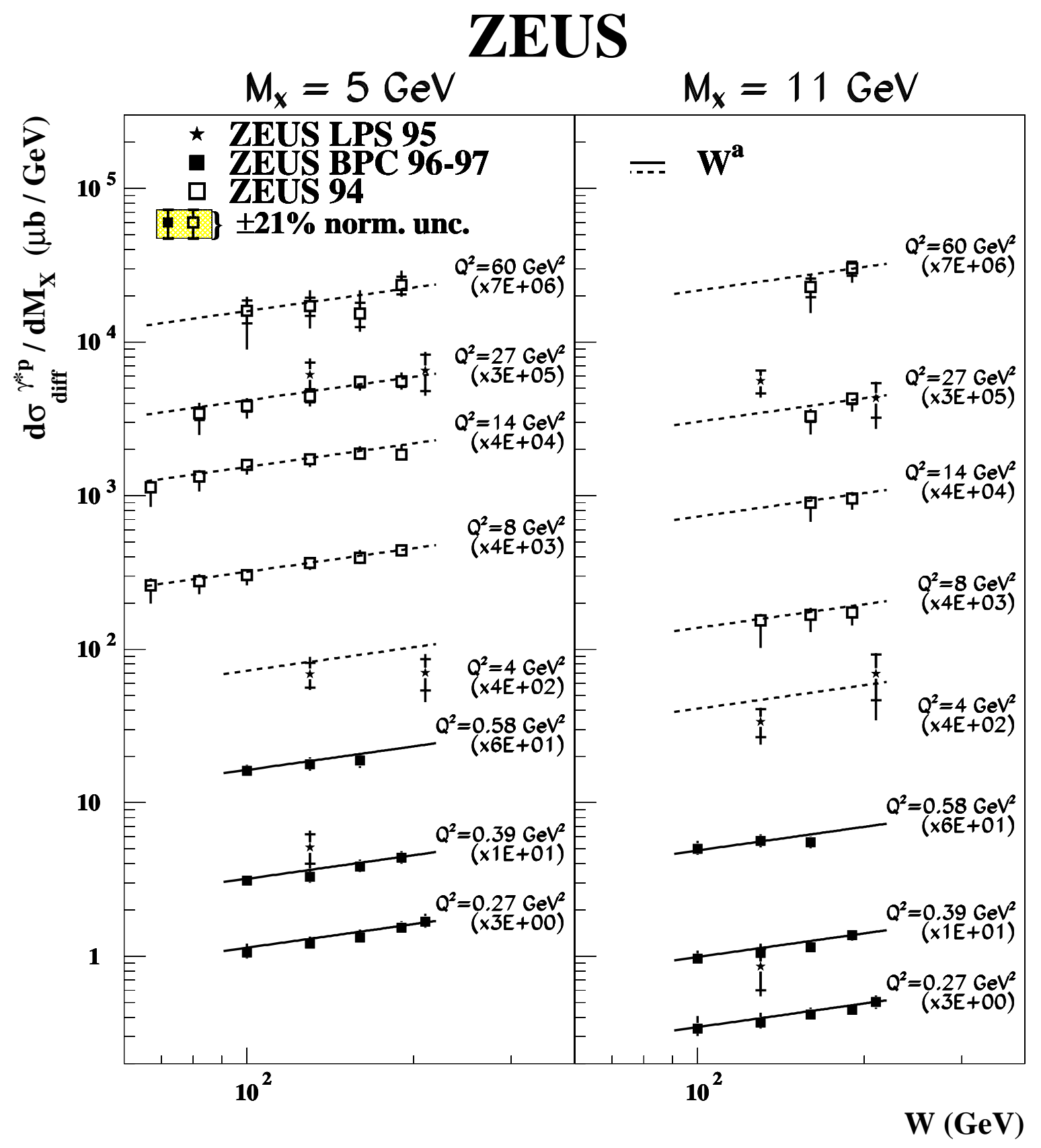

Figure 4: Diffractive cross sections for different $Q^{2}$ and $M_{X}$ values as a function of $W$. The results obtained with the LPS method are shown as stars. The inner bars indicate the size of the statistical uncertainties; the outer bars show the size of the statistical and systematic uncertainties added in quadrature. The low- $Q^{2}$ points obtained with the $M_{X}^{2}$ method (full squares) were corrected for the doubledissociative background; the corresponding $\pm 21 \%$ normalisation uncertainty is not included, but is shown separately as a shaded band. The normalisation uncertainty associated with the luminosity measurement is not shown. The open squares at high $Q^{2}$ are from a previous ZEUS publication [T] and have been corrected for the double-dissociative background using the same estimate as for the low- $Q^{2}$ points, as discussed in the text. The solid lines are the results of the fit to the BPC data described in the text, which also gives a good representation of the higher- $Q^{2}$ data (dashed lines). 


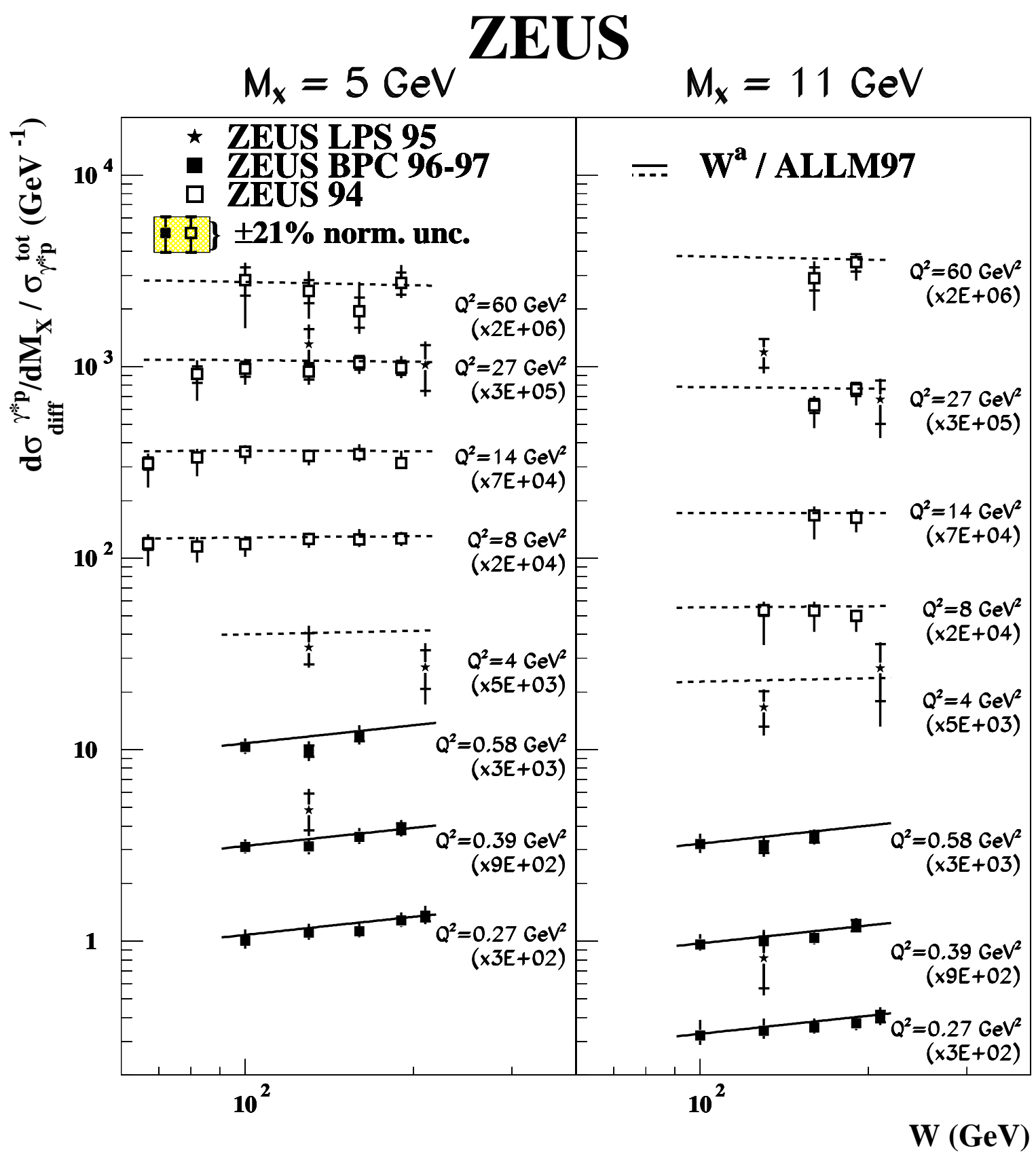

Figure 5: The ratio of the diffractive cross section to the $\gamma^{*} p$ total cross section for different $Q^{2}$ and $M_{X}$ values as a function of $W$. Other details are as in the caption to Fig. 团. The lines denote the fit shown in Fig. 团 divided by the corresponding values of $\sigma_{\mathrm{tot}}^{\gamma^{*} p}$ from the ALLM97 parameterisation. 


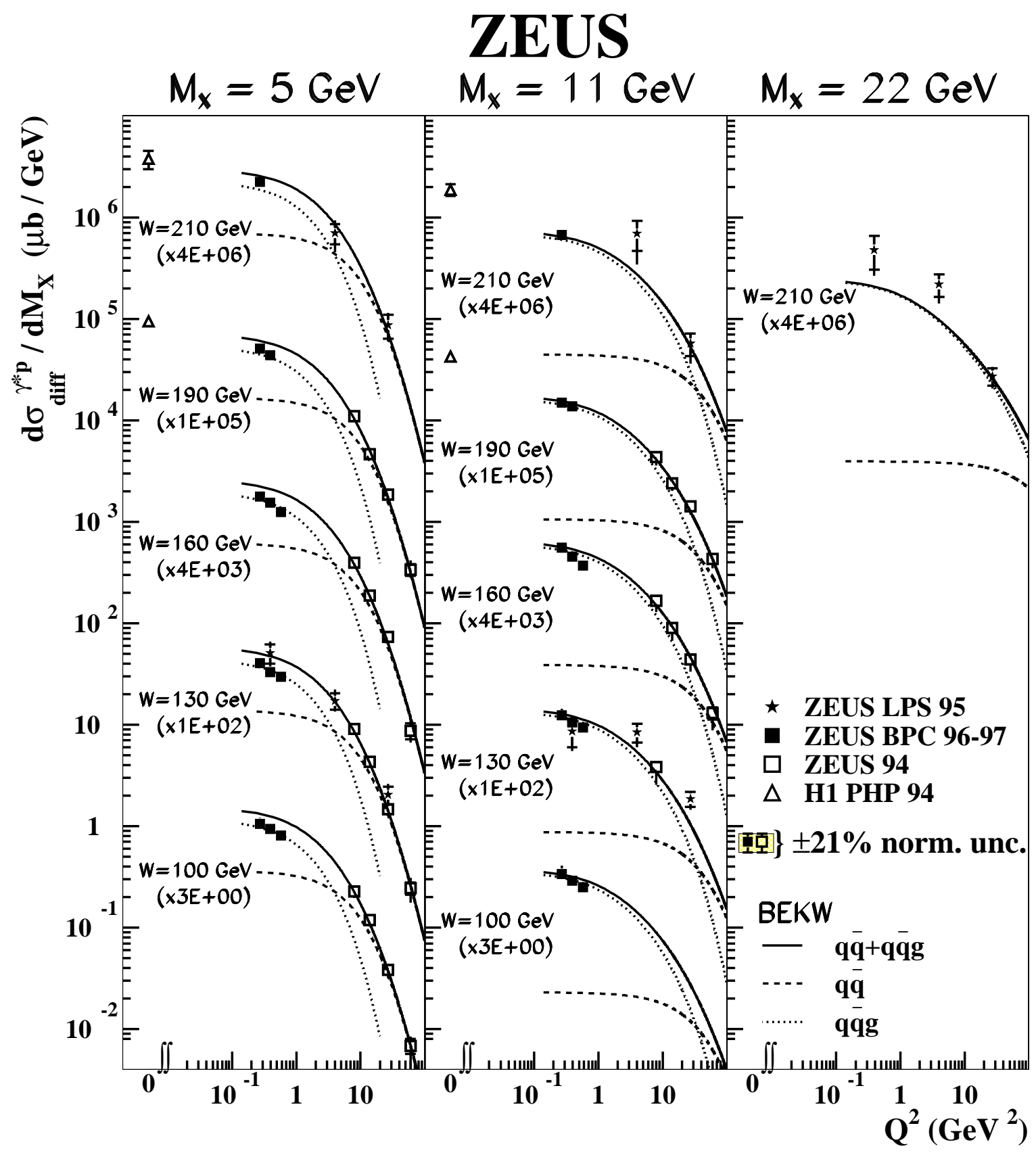

Figure 6: The values of $d \sigma_{\mathrm{diff}}^{\gamma^{*} p} / d M_{X}$ for different $W$ and $M_{X}$ values as a function of $Q^{2}$. Other details are as given in the caption to Fig. $\mathbb{\text { Q }}$. The solid lines are the results of the BEKW parameterisation described in the text; the dotted (dashed) lines are the results of the same parameterisation for the $q \bar{q} g(q \bar{q})$ contribution alone. Note the break in the $Q^{2}$ scale below $\sim 10^{-2} \mathrm{GeV}^{2}$. 


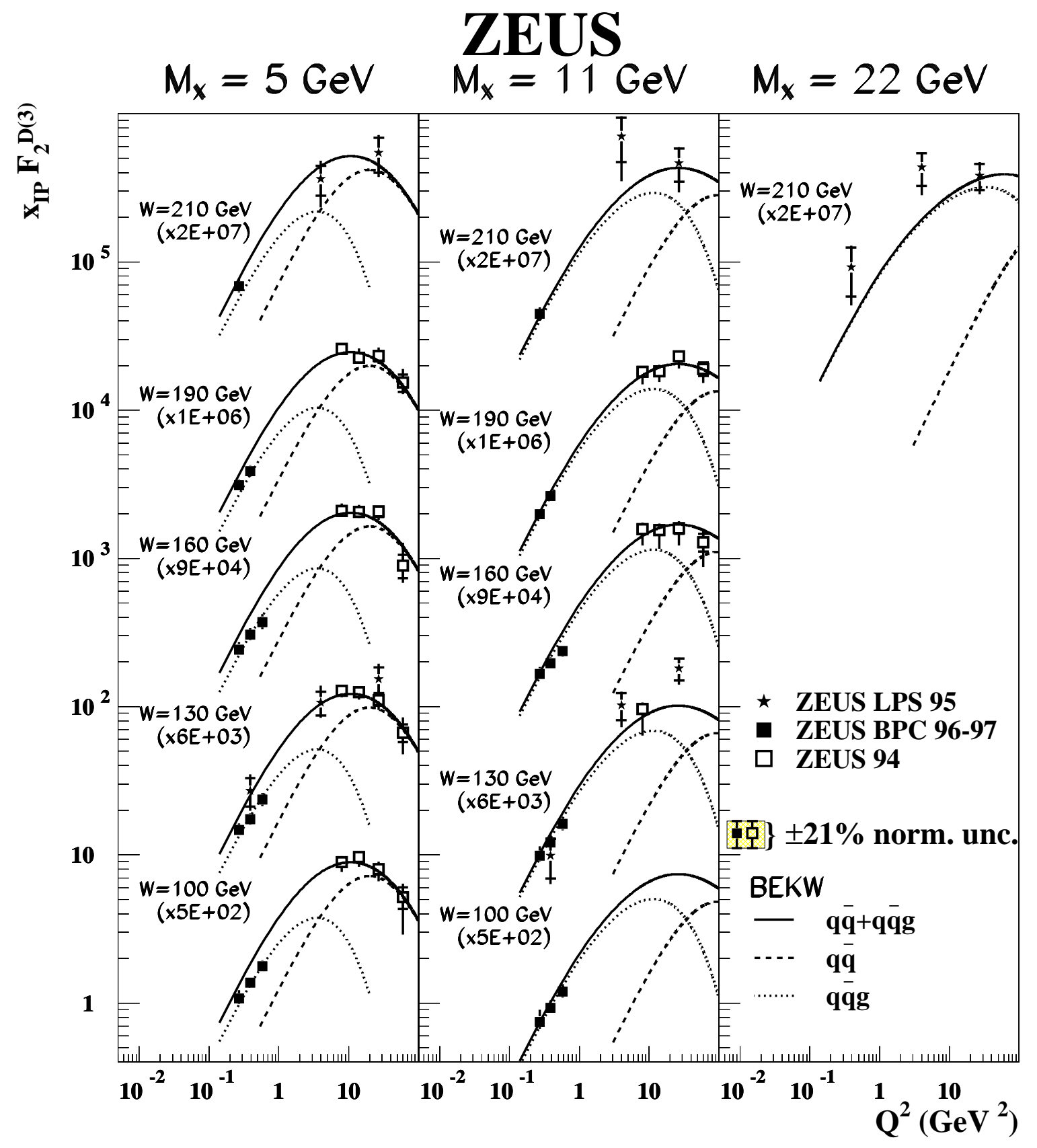

Figure 7: $\quad$ The values of $x_{\mathbb{P}} F_{2}^{D(3)}$ for different $W$ and $M_{X}$ values as a function of $Q^{2}$. Other details are as given in the caption to Fig. 因. The solid lines are the results of the BEKW parameterisation described in the text; the dotted (dashed) lines are the results of the same parameterisation for the $q \bar{q} g(q \bar{q})$ contribution alone. 


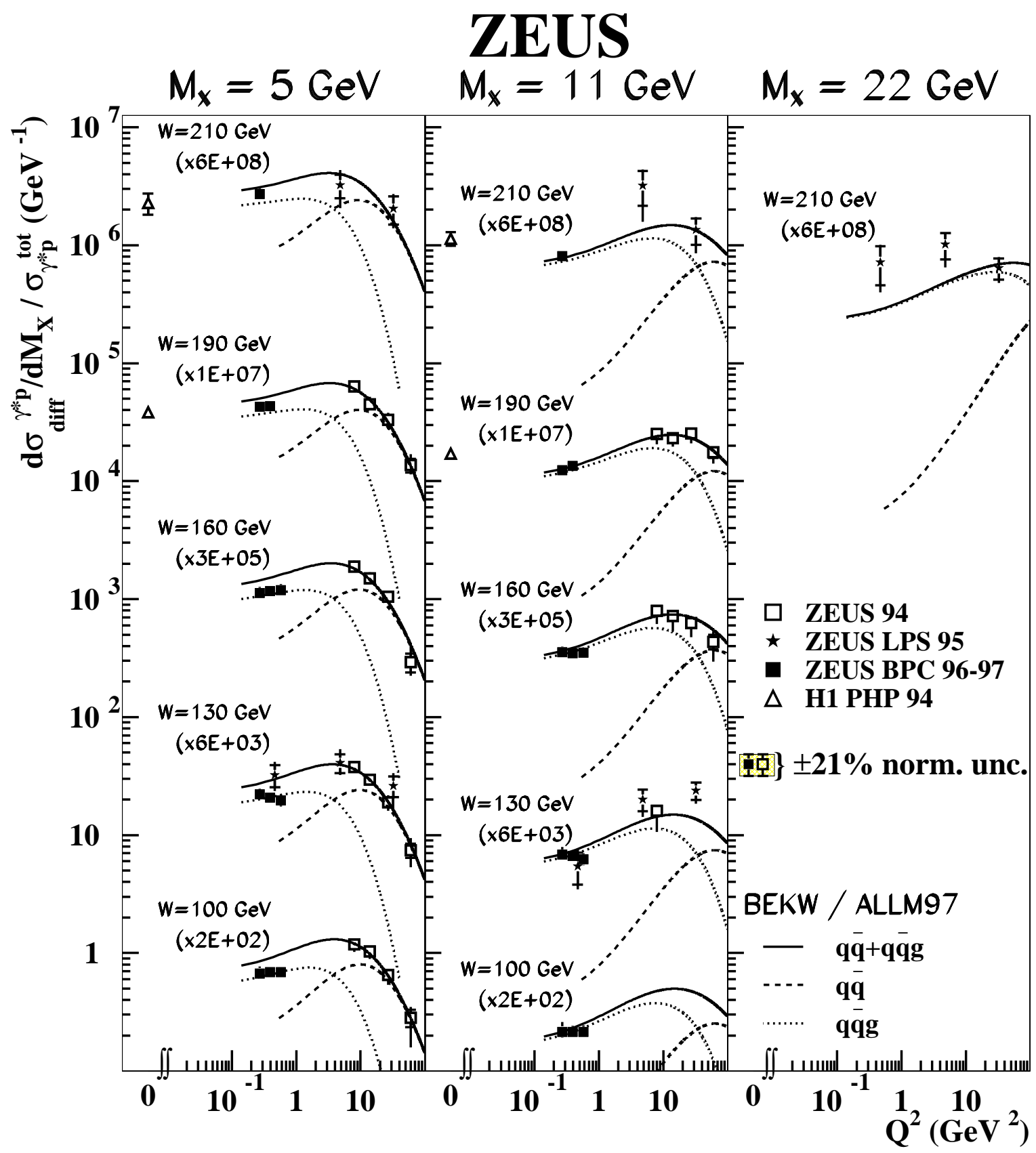

Figure 8: The ratio of the diffractive cross section to the $\gamma^{*} p$ total cross section for different $W$ and $M_{X}$ values as a function of $Q^{2}$. Other details are as given in the caption to Fig. 因. The solid lines are the results of the BEKW parameterisation described in the text, divided by the corresponding $\sigma_{\text {tot }}^{\gamma^{*} p}$ values from the ALLM97 parameterisation; the dotted (dashed) lines are the results of the same parameterisations for the $q \bar{q} g(q \bar{q})$ contribution alone. Note the break in the $Q^{2}$ scale below $\sim 10^{-2} \mathrm{GeV}^{2}$. 